\title{
Klein scattering of spin-1 Dirac-Weyl wave and localized surface plasmon
}

\author{
Hong-Ya Xu, ${ }^{1}$ Liang Huang, ${ }^{2}$ and Ying-Cheng Lai $\odot^{1,3, *}$ \\ ${ }^{1}$ School of Electrical, Computer, and Energy Engineering, Arizona State University, Tempe, Arizona 85287-5706, USA \\ ${ }^{2}$ School of Physical Science and Technology, Lanzhou University, Lanzhou, Gansu 730000, China \\ ${ }^{3}$ Department of Physics, Arizona State University, Tempe, Arizona 85287-5706, USA
}

(Received 15 January 2020; revised 18 January 2021; accepted 17 February 2021; published 26 March 2021)

\begin{abstract}
Localized surface plasmon and Klein tunneling resonances are two phenomena that were previously thought to be unrelated, where the former plays an important role in subwavelength optics while the latter is fundamental to relativistic quantum mechanics and physics of Dirac materials. We develop a rigorous theory for spin-1 DiracWeyl particles, which establishes a striking analogy between the two phenomena and unveils a deep connection between the distinct physical contexts, paving the way for gate-controlled surface plasmon mimetic electronics as well as for realizing localized spoof plasmons in a scope wider than previously thought. A possible experimental scheme is articulated.
\end{abstract}

DOI: 10.1103/PhysRevResearch.3.013284

\section{INTRODUCTION}

Electronic and optical waves share a plethora of phenomena such as interference, diffraction, and resonances. The analogy extends to the classical regime where electron trajectories obeying the principle of least actions correspond to rays in geometrical optics governed by the principle of least time. Exploiting the close analogy between electronics and optics has led to breakthroughs such as photonic crystals for controlling light and the invention of the electron microscope [1-3]. At a fundamental level, the Maxwell's equations can be recast into a matrix wave equation of Hamiltonian form involving spin-1 operators, which resembles the Dirac equation and exposes the quantum spin nature of light [4-6]. On the spin degree of freedom, an analogy exists between propagating surface optical modes at a metal-vacuum interface and the surface electronic states in topological insulators, as predicted by the Dirac equation with a position-dependent, sign-changing mass [7,8]. Recent studies of this analogy shed light on the topological origin and properties of surface Maxwell waves in continuous media [9-12] and other classical waves or collective excitations in different contexts of physics $[13,14]$.

Recent years have witnessed a growing interest in exploring and exploiting the interplay between optical phenomena of light and electronic behaviors in condensed matter systems based on the picture of quasiparticles. For example, in graphene, topological insulators, and Weyl semimetals, electrons can behave as massless particles obeying a linear energy-momentum dispersion relation and are thus reminis-

\footnotetext{
*Ying-Cheng.Lai@asu.edu

Published by the American Physical Society under the terms of the Creative Commons Attribution 4.0 International license. Further distribution of this work must maintain attribution to the author(s) and the published article's title, journal citation, and DOI.
}

cent of photons. In Dirac material systems, demonstrated optical-like phenomena include the electronic analogs of Veselago lens with Klein tunneling-enabled high transparency [15], caustics [16], Mie scattering [17,18], the quantum GoosHänchen effect [19], and the Imbert-Fedorov shift [20]. With the principles of optics, it is possible to realize highly tunable electron optics elements with Dirac fermions for solid-state device applications such as quantum whispering gallery resonators [21], fiberlike electron waveguiding [22,23], and collimator-reflector based quantum switches [24]. The unique properties of Dirac cones and pseudospin in turn can be exploited for applications in electromagnetic wave optics such as the development of photonic Dirac or Weyl metamaterials $[25,26]$.

In this paper, we report an analogy between the localized surface plasmon in optics and Klein scattering resonances in pseudospin-1 Dirac material systems. In particular, we establish a quantitative correspondence between the solutions of pseudospin- 1 waves in the setting of potential scattering and those of Maxwell's equations for scattering from a metallic cylinder. We obtain analytic expressions of the analogous Drude dielectric permittivity as a function of the applied potential height (instead of the intrinsic material-specific parameters), rendering it experimentally tunable. In the regime of small scatterer size, the resonance formulas, line shapes, spatial patterns of the near-field intensities, and flows or currents in both cases bear a remarkable similarity. This finding is surprising because conventionally the Klein effect makes the scalar potential highly transparent $[27,28]$ and therefore provides the underlying mechanism for generating a Veselago lens in Dirac electron optics [29-32]. Our finding offers an approach to realizing surface plasmon mimetic states of spin1 Dirac-Weyl electrons without requiring any signchanging mass or band-gap opening. It also opens up an avenue to generate localized spoof plasmons in the photonic setting of spin-1 Dirac systems through Klein scattering. We also note that in traditional metamaterial physics, the emergence of 
evanescent surface modes requires a complex wave number with physical restrictions such as band-gap opening or a negative permittivity, but our work presents a different route to such modes: They can arise in gapless pseudospin-1 Dirac material systems via the Klein scattering resonance mechanism.

\section{RESULTS}

\section{A. Proof of the analog between Klein scattering resonances and localized surface plasmon modes}

We derive the analogy by exploiting the general setting of spin-1 Dirac-Weyl wave scattering from a circular scalar potential barrier $[17,33]$ and light scattering from a long metallic cylinder with Drude dielectric permittivity in vacuum or in a host dielectric medium. The analogy is established explicitly in terms of closed-form resonance formulas.

We first analyze spin-1 Dirac-Weyl wave scattering, which is governed by the generalized Dirac-Weyl equation

$$
\hat{H} \Psi(\boldsymbol{r})=\left[-i \hbar v_{F} \hat{\boldsymbol{S}} \cdot \nabla+\mathcal{V}(\boldsymbol{r})\right] \Psi(\boldsymbol{r})=E \Psi(\boldsymbol{r}),
$$

in the position representation, where $v_{F}$ is the Fermi velocity, $\Psi=\left[\psi_{1}, \psi_{2}, \psi_{3}\right]^{T}, \hat{\boldsymbol{S}}=\left(\hat{S}_{x}, \hat{S}_{y}\right)$ are the spin-1 matrices, and $\mathcal{V}=V_{0} \Theta(R-r)$ is a scalar potential of finite range of radius $R$. The scattering efficiency, i.e., the ratio of the scattering cross section $\tilde{\sigma}$ to the geometric cross sections, can be used to characterize the process. In the subwavelength regime $R \ll \lambda / 2 \pi$, with $\lambda$ being the wavelength of the incoming spin-1 Dirac-Weyl particles, we finally obtain the scattering efficiency (see Appendix A)

$$
\tilde{\Lambda}_{\text {scat }} \approx \frac{\pi^{2}}{4} \frac{\left(\epsilon_{s}-1\right)^{2}}{\left(\epsilon_{s}+1\right)^{2}+\left(\pi^{2} \rho^{4} / 16\right)\left(\epsilon_{s}-1\right)^{2}} \rho^{3},
$$

with $\rho=k R \equiv E R /\left(\hbar v_{F}\right)$, and

$$
\epsilon_{s}=s_{I} s_{O}\left|\left(E-V_{0}\right) / E\right|,
$$

where the indices $s_{I}=\operatorname{sgn}(E)$ and $s_{O}=\operatorname{sgn}\left(E-V_{0}\right)$ label the energy bands inside and outside the potential barrier, respectively.

In comparison, for scattering of an electromagnetic wave of transverse magnetic (TM) polarization from a metallic cylinder of the same radius $R$, the scattering efficiency for $\rho \ll 1$ is given by [34-36] (Appendix A)

$$
\Lambda_{\text {scat }} \approx \frac{\pi^{2}}{4} \frac{(\epsilon-1)^{2}}{(\epsilon+1)^{2}+\left(\pi^{2} \rho^{4} / 16\right)(\epsilon-1)^{2}} \rho^{3} .
$$

In the Drude approximation, the dielectric permittivity is

$$
\epsilon=1-\frac{\omega_{p}^{2}}{\omega^{2}+\alpha^{2}}+i \frac{\alpha}{\omega} \frac{\omega_{p}^{2}}{\omega^{2}+\alpha^{2}},
$$

where $\omega_{p}$ and $\alpha$ are the plasma and collision frequencies, respectively. Equation (4) characterizes the localized surface plasmon resonance at $\epsilon=-1$, signifying a sign change in the dielectric permittivity across the scatterer interface. Equation (5) gives, in the limit of vanishing collision dissipation rate $\alpha=0$, the surface plasmon resonance frequency as $\omega=\omega_{p} / \sqrt{2}$.

We now compare spin-1 Dirac-Weyl wave scattering with electromagnetic wave scattering. Firstly, Eqs. (2) and (4) reveal a perfect analogy, with $\epsilon_{s}$ in Eq. (2) regarded as the effective dielectric permittivity $\epsilon_{\mathrm{eff}}$ for a spin-1 wave, despite the difference in the underlying physics. Secondly, Eq. (3) indicates that a negative value of $\epsilon_{S}$ is attained through a sign change in the band indices $s_{I} s_{O}=-1$ with the Klein scattering of particle-antiparticle transmutation across the potential barrier boundary, while that of $\epsilon$ based on Eq. (5) is responsible for the optical response of metals below the plasma frequency. The former leads to the resonant energy or frequency $E=V_{0} / 2$, which is tunable by varying the external potential height $V_{0}$, while the latter is limited to the material specified plasma frequency. For some small scatterer size, e.g., $\rho=0.1$, numerical calculations confirm the similarity in the line shape (spectrum profile) of the Klein scattering resonance of spin-1 Dirac-Weyl waves and the well-known localized surface plasmon resonance in light scattering, as shown in Figs. 1(a) and 1(b), respectively. From the spatial patterns of the intensity distributions of the spinor wave function and magnetic field in the middle (a dipole resonance) and right (a quadruple resonance) panels, we observe an almost exact correspondence between the Klein scattering resonance modes and the excited localized surface plasmon modes.

The physical insights into the scattering efficiency associated with surface plasmon and its connection to the three-band Dirac model are as follows. The gapless spin-1 Dirac-Weyl spectrum possesses two linear bands and a flat band. Recalling that surface plasmon arising at a dielectricmetal interface can be attributed to the interplay between the electromagnetic wave and plasma oscillations in the metal, we have that the two linear bands resemble the conical dispersion of light, while the flat band corresponds to the dispersionless plasma frequency. The applied scalar potential controls the flat-band position and induces a sign change in the band index across the potential boundary via a simultaneous shift in the Dirac point. This plays an analogous role to that of the required metal element in exciting localized surface plasmons from light scattering, which determines the plasma frequency and is responsible for the necessary sign change in the real part of the dielectric function across the dielectric-metal interface. In particular, the analogous plasma frequency is given by $\tilde{\omega}_{p}=V_{0} / \hbar$, below which $\left(E / \hbar=\tilde{\omega}<\tilde{\omega}_{p}\right)$ localized surface-plasmonlike modes arise from the Klein scattering resonance about $\tilde{\omega}=\tilde{\omega}_{p} / 2$.

We note that in pseudospin-1 systems, the Berry phase around the Dirac point is zero or $2 \pi$. This is a general indication of the presence of backscattering and thus an anti-Klein tunneling behavior. It can be used to understand, for example, transport in bilayer graphene. The case of a pseudospin-1 Dirac system is subtle because, when subject to a one-dimensional scalar potential step, it exhibits the phenomenon of super-Klein tunneling, i.e., the potential becomes more transparent than that for pseudospin-1/2 systems. However, backscattering generally arises from the two-dimensional scalar potential scattering due to the zero or $2 \pi$ Berry phase argument.

The analogy exemplified in Fig. 1 is not simply another case of anti-Klein tunneling, but demonstrates the emergence of peculiar surface modes in the Klein tunneling regime. It is counterintuitive and requires an understanding beyond the Berry phase argument, as the presence of backscattering 

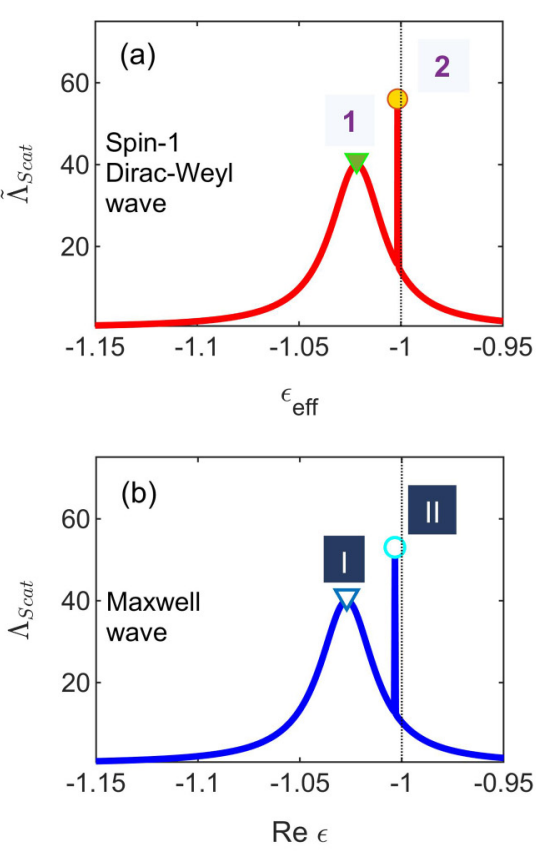

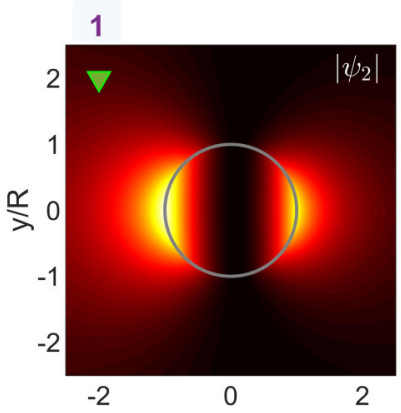

I: dipole

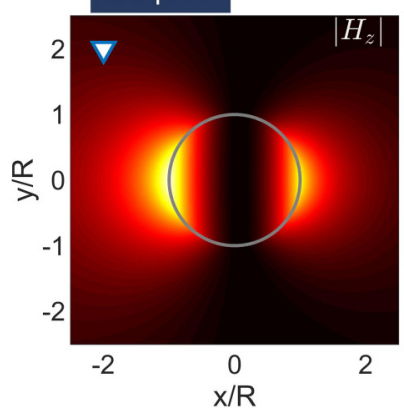

2

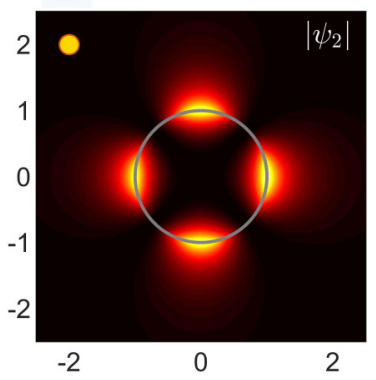

II: quadrupole

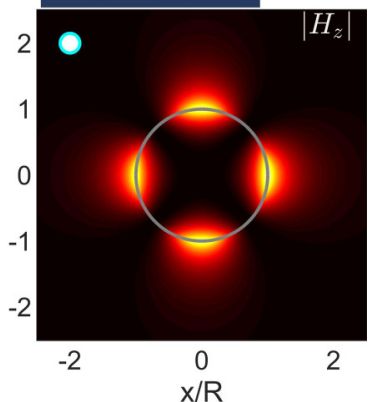

FIG. 1. Analogy between surface resonant modes in Klein scattering of a spin-1 Dirac-Weyl wave and surface plasmon modes in optics. Plotted is the scattering resonance line shape for (a) a massless spin-1 wave and (b) light scattering by a metallic wire with zero collision dissipation rate $\alpha=0$, for scatterer size $\rho=0.1$. Right panels show the spatial patterns of the corresponding resonant modes as indicated in (a) and (b), where $\psi_{2}$ is the second component of the spin- 1 wave function. For light scattering, $H_{z}$ denotes the magnetic field.

associated with the zero or $2 \pi$ Berry phase is only marginally relevant to the occurrence of the surface modes. This is because the phenomenon does not arise spontaneously in bilayer graphene where the Berry phase is $2 \pi$ in the Klein tunneling regime but requires an external magnetic field or some topological origin in gapped two-dimensional (2D) Dirac systems. The contribution of our work is an understanding of such anti-Klein tunneling surface modes through an analogy with the well-known localized surface plasmon resonance modes in optics. In particular, we have analytically demonstrated the analogy in terms of the closed-form resonance formulas [Eqs. (2) and (4)] obtained from the paradigmatic settings. Furthermore, as demonstrated below, revisiting the Hamiltonian form of the Maxwell's equations, we have elucidated the physical idea underlying the striking analogy between the Poynting and spin-1 Dirac currents. Importantly, we find that the close analogy lies in the correspondence of boundary conditions for Klein tunneling of a spin-1 Dirac wave and localized surface mode excitations in optics.

\section{B. Dirac currents and Poynting vectors}

To further establish the analogy between the two physically distinct scattering processes, we analyze the underlying flow patterns for Figs. 1(a) and 1(b) by calculating the currents of the spin-1 Dirac-Weyl waves and the total Maxwellwave-defined Poynting vectors, as shown in Fig. 2. The resemblance is striking in terms of the phase information and the topological property of vortices as well as the saddle structure embedded in the flows. (Extended and more detailed comparisons between the Dirac currents and Poynting vectors reinforcing the analogy with varying scatterer size and across a particular resonance are presented in Appendix B.) Heuristic insights into the correspondence can be obtained by analyzing the Hamiltonian form of the Maxwell's equations. For a monochromatic electromagnetic wave of frequency $\omega$ described by complex electric and magnetic field amplitudes $\boldsymbol{E}(\boldsymbol{r})=\left(E_{x}, E_{y}, 0\right)$ and $\boldsymbol{H}(\boldsymbol{r})=$ $\left(0,0, H_{z}\right)$, the Maxwell's equations in an isotropic, lossless, and homogeneous optical medium characterized by the realvalued permittivity $\epsilon$ and permeability $\mu$ are $\nabla \times \boldsymbol{H}(\boldsymbol{r})=$ $-i \epsilon \omega \boldsymbol{E}(\boldsymbol{r})$ and $\nabla \times \boldsymbol{E}(\boldsymbol{r})=i \mu \omega \boldsymbol{H}(\boldsymbol{r})$, which can be expressed as a matrix equation

$$
\frac{1}{\sqrt{2}}\left(\begin{array}{ccc}
0 & k_{-} & 0 \\
k_{+} & 0 & k_{-} \\
0 & k_{+} & 0
\end{array}\right) \psi=\omega\left(\begin{array}{ccc}
\epsilon & 0 & 0 \\
0 & \mu & 0 \\
0 & 0 & \epsilon
\end{array}\right) \psi,
$$

where $k_{ \pm} \equiv k_{x} \pm i k_{y}$ and

$$
\psi \equiv\left[-\left(E_{x}-i E_{y}\right) / \sqrt{2}, i H_{z},\left(E_{x}+i E_{y}\right) / \sqrt{2}\right]^{T} .
$$

Using the transformation

$$
\Phi=\Lambda \psi=\left[\phi_{1}, \phi_{2}, \phi_{3}\right]^{T}=\left(\begin{array}{c}
-\sqrt{\epsilon / 2}\left(E_{x}-i E_{y}\right) \\
i \sqrt{\mu} H_{z} \\
\sqrt{\epsilon / 2}\left(E_{x}+i E_{y}\right)
\end{array}\right),
$$

with $\Lambda=\operatorname{diag}(\sqrt{\epsilon}, \sqrt{\mu}, \sqrt{\epsilon})$, we obtain

$$
(\mu \epsilon)^{-1 / 2} \hat{\boldsymbol{S}} \cdot \boldsymbol{k} \Phi=\omega \Phi,
$$

where $\boldsymbol{k}=\left(k_{x}, k_{y}\right)$. Interpreting $\Phi$ as the "photon wave function," we have the optical counterpart of the local spin-1 Dirac current as

$$
1 / \sqrt{\mu \epsilon} \Phi^{\dagger} \hat{\boldsymbol{S}} \Phi=2 \operatorname{Re}\left[\boldsymbol{E} \times \boldsymbol{H}^{*}\right]
$$



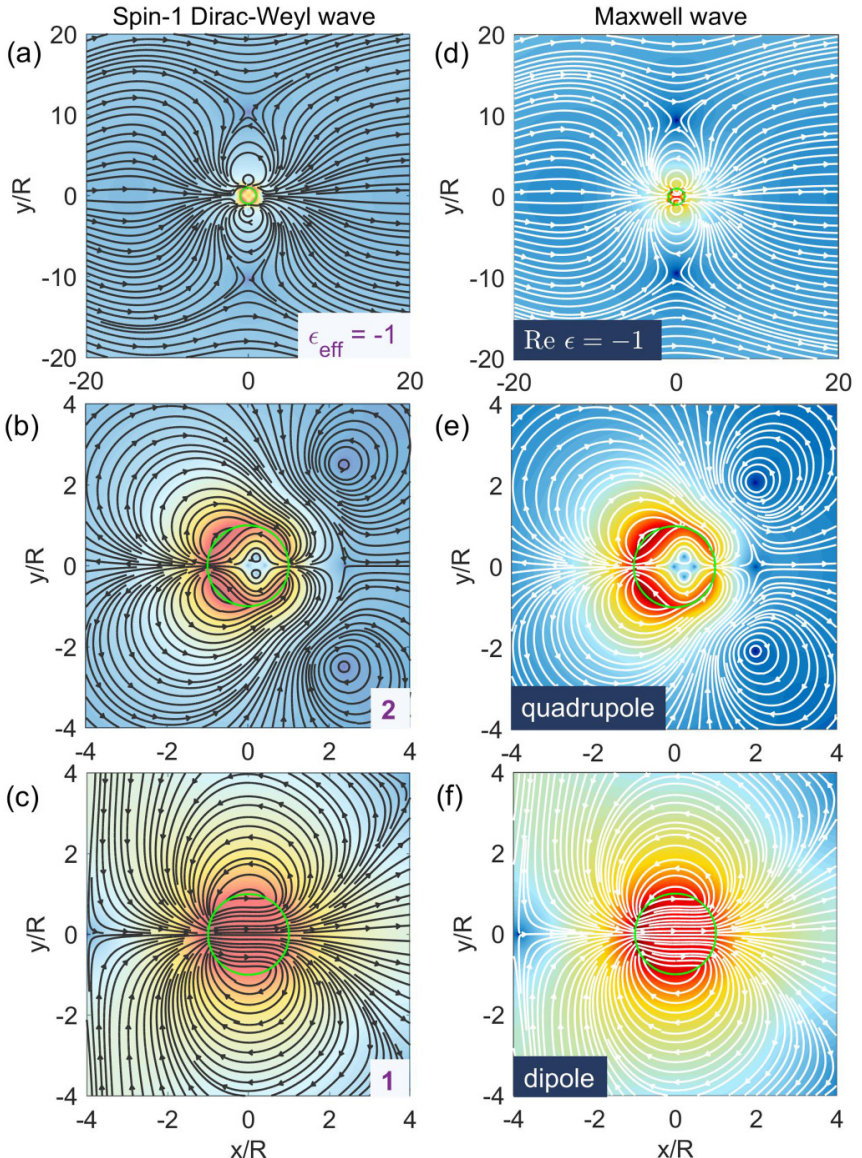

(e)
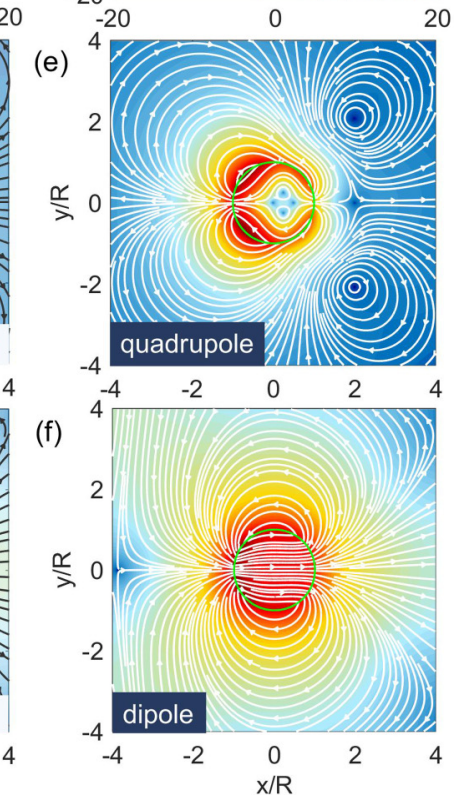

FIG. 2. Comparison in terms of local spin-1 Dirac currents and Poynting vectors. (a)-(c) Spatial distributions of the local currents (black arrowed streamlines) of spin-1 Dirac-Weyl wave scattering by a scalar potential barrier in the setting of Fig. 1(a). (d)-(f) The corresponding results for the Poynting vectors (white arrowed streamlines) and color-coded contour maps of their magnitude for electromagnetic wave scattering from a small (subwavelength) metallic cylinder in the setting of Fig. 1(b). A more extensive and detailed comparison between the Dirac currents and Poynting vectors is presented in Appendix B.

which is nothing but the exact time-averaged Poynting vector (energy flux) [37,38]:

$$
\langle\boldsymbol{P}\rangle=(c / 8 \pi) \operatorname{Re}\left[\boldsymbol{E} \times \boldsymbol{H}^{*}\right] .
$$

The remarkable similarity in the spatial patterns of the Dirac current and the Poynting vector field thus holds at a deeper level involving sophisticated phase information and topological structures. The analogies uncovered in the scattering line shape, the spatial intensity profile of the resulting resonance modes, and the flow or current pattern are exact in the subwavelength regime $\rho \ll 1$ but tend to break down for $\rho>1$. (A detailed account of the validity and breaking down of regimes is presented below.)

The key to the common physics underlying the two contexts lies in the boundary conditions. In the spin-1 Dirac system, the wave function is a three-component Dirac-Weyl spinor $\Phi=\left[\phi_{1}, \phi_{2}, \phi_{3}\right]^{T}$. We find that, crossing a potential boundary with the outward unit normal $\hat{e}_{n}=(\cos \theta, \sin \theta)$, the conservation of the normal component of the spin-1 Dirac

current stipulates that $\phi_{2}$ and $\left(\phi_{1} e^{i \theta}+\phi_{3} e^{-i \theta}\right)$ must be continuous, which implies a discontinuity in $\left(\phi_{1} e^{i \theta}-\phi_{3} e^{-i \theta}\right)$. Likewise, in electromagnetic wave scattering, boundary conditions play an essential role in exciting surface plasmon modes and generating a surface charge distribution leading to a discontinuity in the electric field component normal to the surface and field enhancement near the surface. These features reflect the bound nature of surface plasmon modes [39]. Using the recast spinor form of the electromagnetic field [Eq. (7)] in the tangential-normal coordinates

$$
\Phi=\left[-\sqrt{\epsilon / 2}\left(E_{n}-i E_{t}\right) e^{-i \theta}, i \sqrt{\mu} H_{z}, \sqrt{\epsilon / 2}\left(E_{n}+i E_{t}\right) e^{i \theta}\right],
$$

we have that the conservation of the spin-1 Dirac current requires continuity of the tangential field components $\left(H_{z}, E_{t}\right)$ at the boundary and a discontinuity in the electric field normal to the boundary $\left(E_{n}\right)$, which are the boundary conditions enabling excitations of surface plasmon in optics. This subtle and fundamental connection between scattering of spin-1 Dirac-Weyl waves and light scattering with surface plasmonic resonance leads to the analogy uncovered in this paper and is the underlying reason for the striking similarity between the resonance formula for the Klein scattering efficiency [Eq. (2)] and that for the localized surface plasmon resonance [Eq. (4)].

A property associated with the excitation of localized surface plasmons is near-field enhancement from a subwavelength confinement with quality strongly dependent on the scatterer geometry [40]. For example, a metallic dimer can be used to achieve strong field enhancement in the center of the gap for small separations due to the strong coupling between the individual plasmons (mode hybridization) [41], with applications in photovoltaics, enhancement of lightmatter interaction, and miniaturization of optical devices [42-44]. Can similar features be expected from the Klein scattering resonances of spin-1 Dirac-Weyl waves? Here, we consider two common dimer configurations, disk and bowtie [insets of Figs. 3(a) and 3(b), respectively], and develop an efficient numerical method to solve the scattering problem (Appendix C). Analogous to the local electric field enhancement of a metallic dimer [45], we use the square of the first or third spin-1 Dirac-Weyl wave function component amplitude at the center of the dimer $\boldsymbol{r}_{\mathbf{0}}$, e.g., $\left|\left\langle\boldsymbol{r}_{\mathbf{0}} \mid \psi_{1}\right\rangle\right|^{2}$, as a function of $k R$ to characterize the near-field intensity enhancement spectra, as shown in Fig. 3. Similar to localized surface plasmons in optics $[45,46]$, the plasmonic effect associated with the field enhancement accompanied by a frequency red shift with a decrease in the separation $d$ can be seen in Figs. 3(a)-3(c), where a mimicking bonding dipole type of plasmon hybridization emerges, as shown in Fig. 3(d).

\section{Relation to experiments}

We articulate an experimental test of our finding through a two-dimensional electronic Lieb lattice [47,48]. To validate and detect the localized plasmon mimetic spin-1 Dirac surface modes, we consider a two-terminal transport system of a waveguide geometry with a short-range potential barrier type of scatterer, as sketched in Fig. 4(a). The associated bulk spin-1 Dirac spectrum is shown in Fig. 4(b). Interference between the waveguiding channels and individual cavity confinement mode would give rise to a dip in the background 
(a)

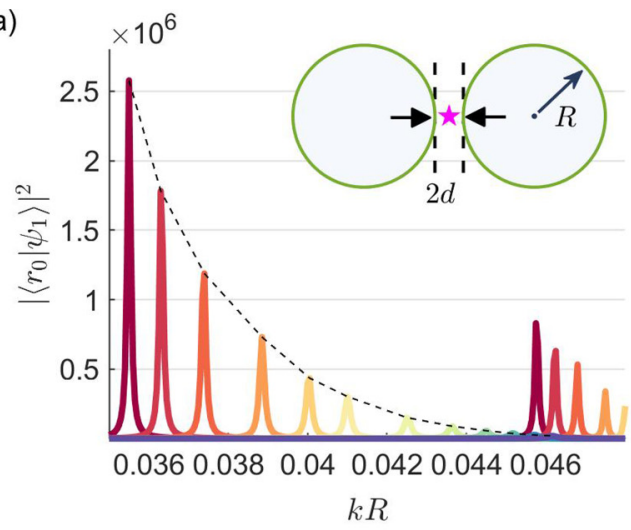

(c)

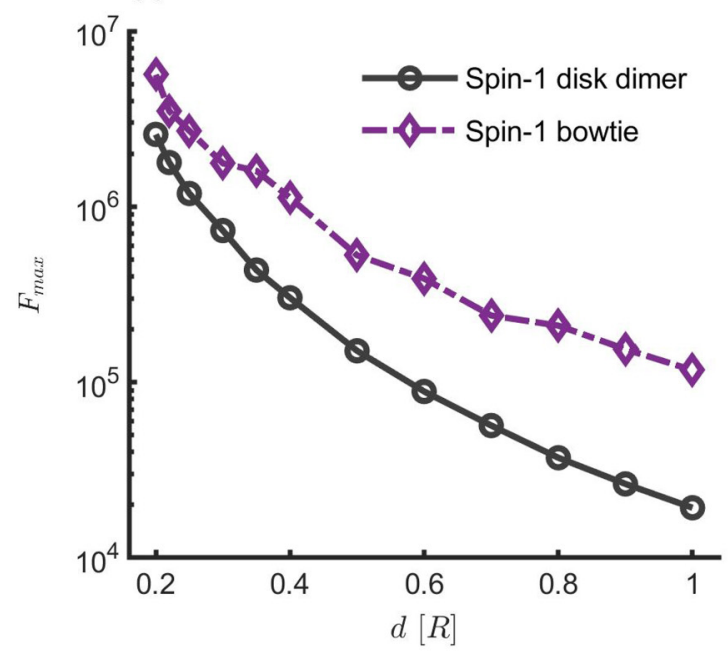

(b)

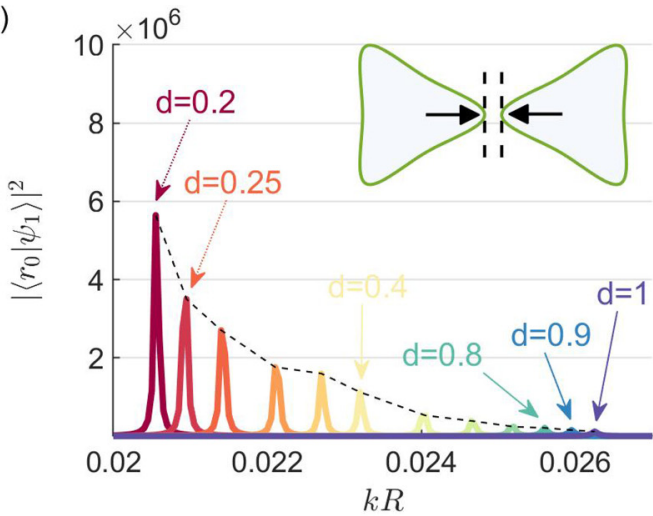

(d)

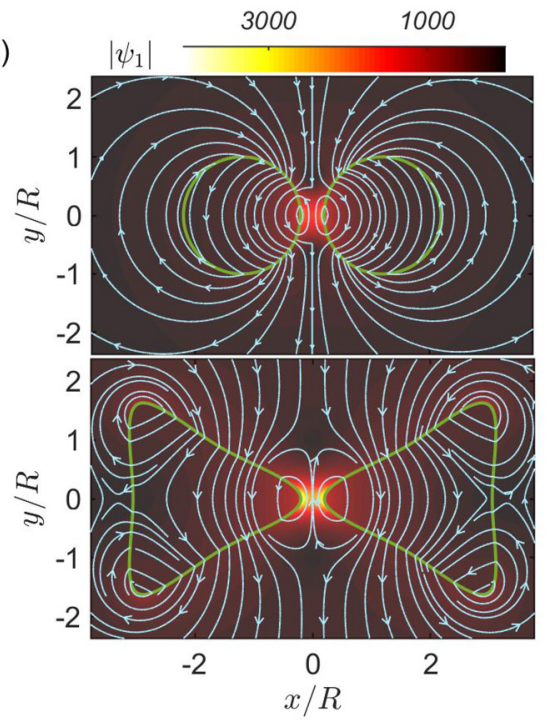

FIG. 3. Field enhancement analog through mode hybridization of coupled Klein scattering. Shown are the scattering resonance spectra resolved by the near-field intensity at the center of a spin-1 Dirac (a) disk dimer and (b) bowtie dimer for different amounts of gap separation $d[R]$ (indicated by different colors). (c) Maximum field intensity at the gap center as a function of gap separation. (d) Real-space amplitude distribution and local Dirac flows at resonances for $d=0.2 R$.

conductance plateau, as shown in Fig. 4(c) for potential height $V_{0} R / \hbar v_{F}=0.6$. Compared with the continuum model based scattering efficiency plot in Fig. 4(d) for an incident plane wave, we obtain a good agreement with our theoretical prediction of the dipole-plasmon-like resonance about $k R=0.3$ in the Klein scattering and subwavelength $(\lambda \approx 24 R)$ regime. The resulting real-space local Dirac current flow [inset of Fig. 4(c)] can be measured via the state-of-the-art quantum imaging technique with nitrogen-vacancy center based sensors [49]. We note that the realizations of artificial photonic and acoustic materials hosting the spin-1 spectrum [50-53] provide more diverse experimental settings to validate our findings.

\section{Breakdown of the analogy between Dirac spin-1 and surface plasmon physics beyond the subwavelength regime}

For inhomogeneous systems with Klein scattering and localized surface plasmon, we have demonstrated that the analogy between Dirac spin-1 and surface plasmon physics is almost perfect but in the subwavelength regime $\rho=k R \ll 1$ (e.g., in optics), where the spatial scale of the scatterer $R$ is much smaller than the incident wavelength $2 \pi / k$ (cf. Fig. 2 and Appendix B). (Figure 8 in Appendix B shows that characteristic differences between electromagnetic and spin- 1 wave scattering will emerge when $k R$ increases to move the systems beyond the subwavelength regime.) The analogy between Dirac spin-1 and surface plasmon physics breaks down for $k R \gtrsim 1$.

Understanding the conditions under which the analogy breaks down is particularly relevant from the point of view of experimental implementation, especially when considering the effectiveness of the Dirac Hamiltonian in the low-energy regime. To provide further evidence to support the analogy, we have carried out computations of an experimentally viable setting of Dirac spin-1 physics: the tight-binding Lieb lattice. A detailed comparison between the current patterns from the spin-1 lattice system and continuum model and those of the Poynting vector from electromagnetic wave scattering is presented in Fig. 5. It can be seen that the similarities in the current patterns among the three cases in the subwavelength regime are striking, providing further support for the uncovered analogy between Dirac spin-1 and surface plasmon physics. It can also be seen that beyond the subwavelength regime, small differences violating the analogy begin to emerge. 


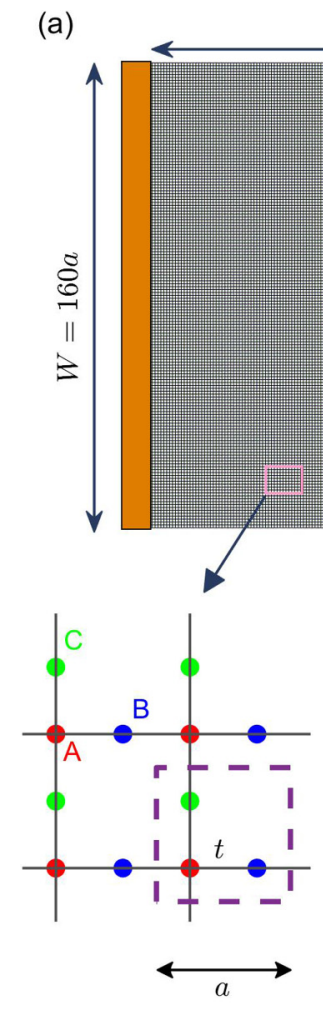

$L=160 a$

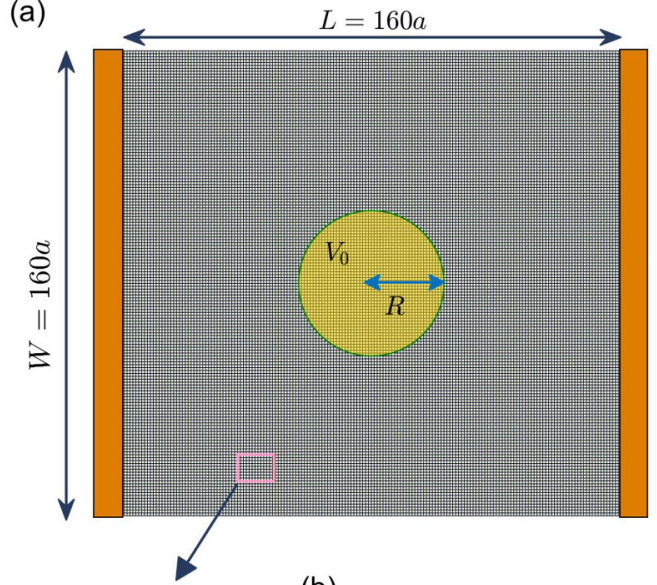

(b)

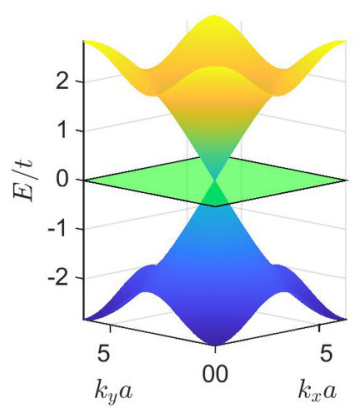

(c)

(d)

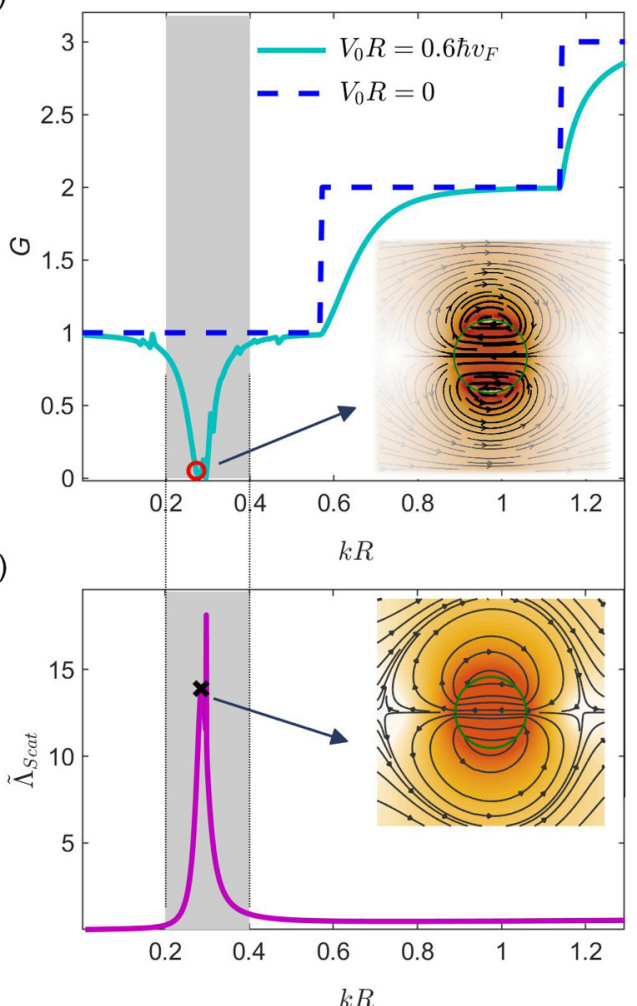

FIG. 4. Validation of spin-1 Dirac physics in an electronic Lieb lattice and signatures in a typical transport measurement. (a) A two-terminal transport device with an electrostatically gated circular region of radius $R$ placed at the center of an electronic Lieb lattice sheet. (b) Bulk energy spectra of the lattice. (c) Conductance as a function of the effective size $k R$ of the scatterer. (d) Scattering efficiency vs $k R$ calculated from the continuum spin-1 Dirac-Weyl model for comparison purposes. The insets of (c) and (d) are spatial patterns of the associated local Dirac current flows at the resonances indicated. The calculations are based on experimentally feasible system parameter values: $a=2.66 \mathrm{~nm}, t=0.1 \mathrm{eV}$, and $v_{F}=3.5 \times 10^{14} \mathrm{~nm} / \mathrm{s}$.

\section{E. Effect of next-nearest-neighbor hopping process}

The analogy or correspondence was established based on the general continuum spin-1 Dirac model and demonstrated using lattice models. The validity of the analogy is limited by how well such a spin-1 Dirac model is realized or simulated in experimental settings, e.g., electronic or photonic Lieb lattices or any other relevant synthetic lattices or materials with the spin-1 Dirac-cone band structure.

In Ref. [47], it was noted that the ratio between the nextnearest-neighbor hopping and the nearest-neighbor hopping is $t^{\prime} / t \sim 1 / 3$, which is relatively large. We thus calculate the effect of the next-nearest-neighbor hopping $t^{\prime}$ on the surface plasmon mode analog via the scalar potential scattering mechanism in the Klein tunneling regime. The result is shown in Fig. 6. It can be seen that for $t^{\prime} / t=1 / 3$, the bulk spectrum flat band curls up beyond the energy scale considered and the phenomenon disappears. However, this does not mean that the analogy breaks down, for the following reasons. First, the analogy or correspondence is established for the spin-1 Dirac systems. Second, the dramatically deformed flat band makes the effective low-energy spin-1 Dirac description inaccurate. When $t^{\prime} / t$ is finite and relatively small so that the spin-1 Dirac Hamiltonian approximation holds, the localized surface plasmon analog is valid. For a perturbed spin-1 Dirac model incorporating the effect of $t^{\prime}$, a closed-form formula such as Eq. (2) is not available.
We note that for an experimentally realized photonic Lieb lattice setting, the next-nearest-neighbor hopping energy is negligible [51]. In this case, our tight-binding Lieb lattice based calculations are relevant.

\section{F. Nonexistence of the analogy in spin-1/2 systems}

Does the analogy arise in spin- $1 / 2$ systems? To address this question, we note that the analogy involves three key physical ingredients: (1) Klein tunneling associated with a sign change in the band index, which is analogous to the sign change in the real part of the dielectric function associated with the energy flow across the interface, (2) the presence of a flat band that plays the role of the dispersionless frequency of surface plasmon excitation as the result of the interplay with the linear dispersion of light, and (3) the Dirac current as the counterpart of the Poynting vector with equivalent boundary conditions. Spin-1/2 Dirac-cone systems such as graphene do not meet conditions (2) and (3), so the analogy between Klein scattering and localized surface plasmon does not arise.

To provide concrete support, we have carried out calculations for the case of a hexagonal lattice with the gapless spin-1/2 Dirac cone. The system has the same hexagonalshaped Brillouin zone. To provide further support that the analogy arises for a spin-1 Dirac system, we have also considered an alternative realization of the spin-1 system (near 


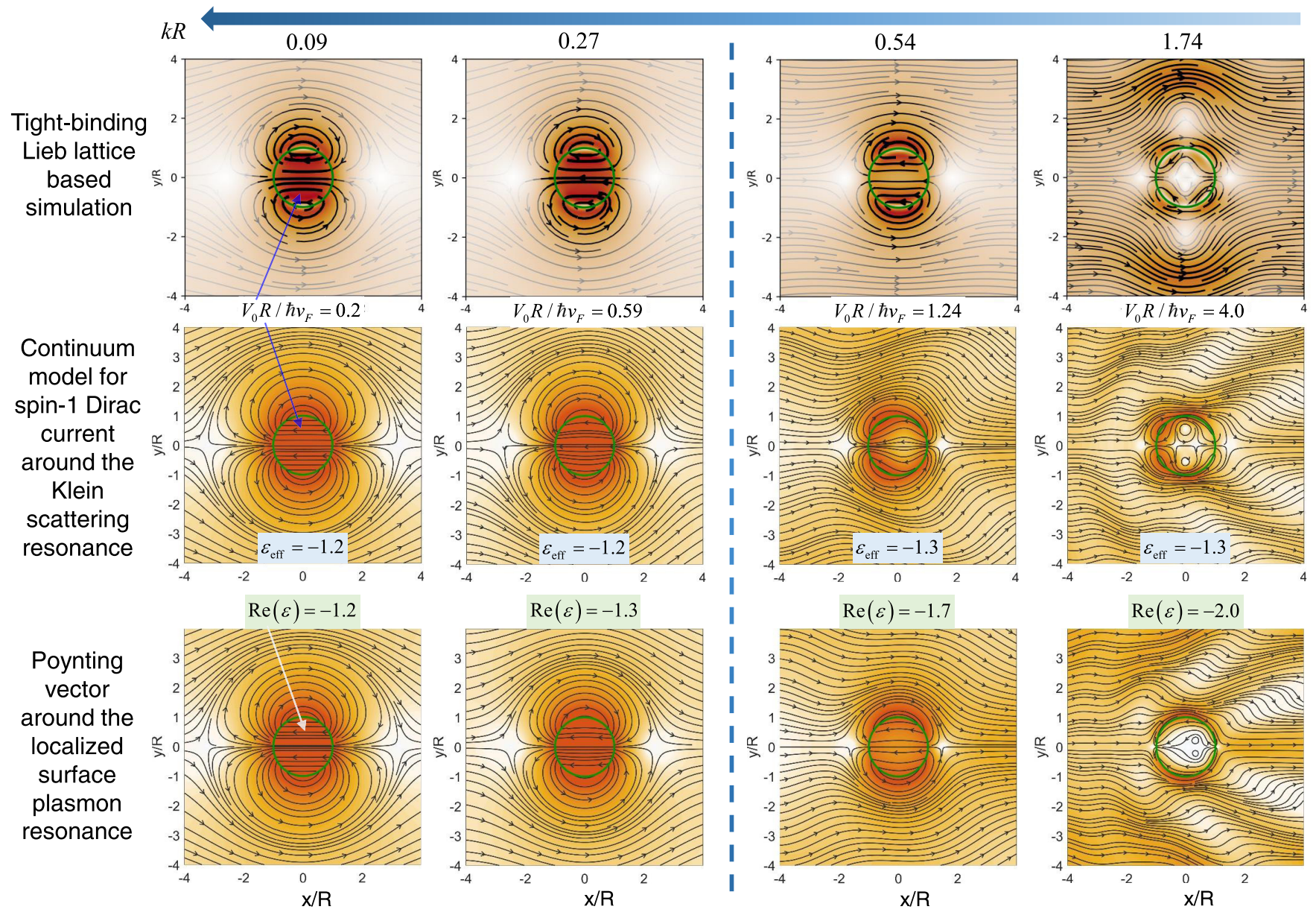

FIG. 5. Comparison of the currents associated with an electromagnetic wave and spin-1 Dirac wave. The top panels show the current from a tight-binding Lieb lattice system with a gapless spin-1 Dirac-cone type of low-energy bulk spectrum from an experimentally viable two-terminal setup illustrated in Fig. 4(a), the middle panels display the spin-1 Dirac current in the continuum model, and the bottom panels demonstrate the Poynting vector associated with electromagnetic wave scattering. The similarities among the three cases in the subwavelength regime are striking. Beyond the subwavelength regime, small differences violating the analogy begin to emerge.

the zone corner $\mathbf{K}$ and $\mathbf{K}^{\prime}$ points): a generalized dice lattice. Representative results are presented in Fig. 7, providing further support for the analogy and the physical conditions under which it arises.

We note that for gapless spin-1/2 Dirac-cone systems, it has been known [30] that Klein tunneling enables an unimpeded penetration through high potential barriers and thus makes it impossible to pinch off the conductance or current effectively. This behavior can be seen from the left panels of Fig. 7. This "expected" behavior does not arise in spin-1 Dirac systems. The analogy between spin-1 Dirac and surface plasmon physics that we have uncovered has thus revealed a different aspect of Klein tunneling physics, due to the coexistence of a flat band and a Dirac-cone structure that is characteristic of spin-1 systems.

Generalizing the predicted analogy to other types of systems with a similar coexistence of Dirac cones and a flat band, e.g., twisted layered graphene systems [54] and Dirac-like semimetals with three-component quasiparticles $[55,56]$, remains completely open. Our finding opens up a new possibility of research for both electronics and (subwavelength) optics, in accordance with the recent surge of interest in developing Dirac materials beyond graphene as well as unconventional photonic Dirac-cone metamaterials.

\section{DISCUSSION}

We have uncovered a striking analogy between localized surface plasmon modes in optics and Klein scattering resonances of spin-1 Dirac-Weyl waves. Despite the distinct underlying physics, the Klein scattering resonances mimic all aspects of the localized surface plasmon modes, indicating a deep connection between the two contexts. We have presented a rigorous demonstration of this analogy based on the Mie scattering theory. At a fundamental level, the finding reveals a unique aspect of the Klein paradox as a mechanism for generating evanescent localized surface plasmons. This is counterintuitive because conventional wisdom stipulates that Klein tunneling enables an unimpeded transmission through potential boundaries via nonevanescent waves, implying that it is not possible to create surface modes through the Klein tunneling mechanism [21,30,31]. With respect to applications, the finding has implications in both electronics and optics with the advent of emerging electronic or photonic spin-1 Dirac-cone metamaterials. For instance, it opens an 

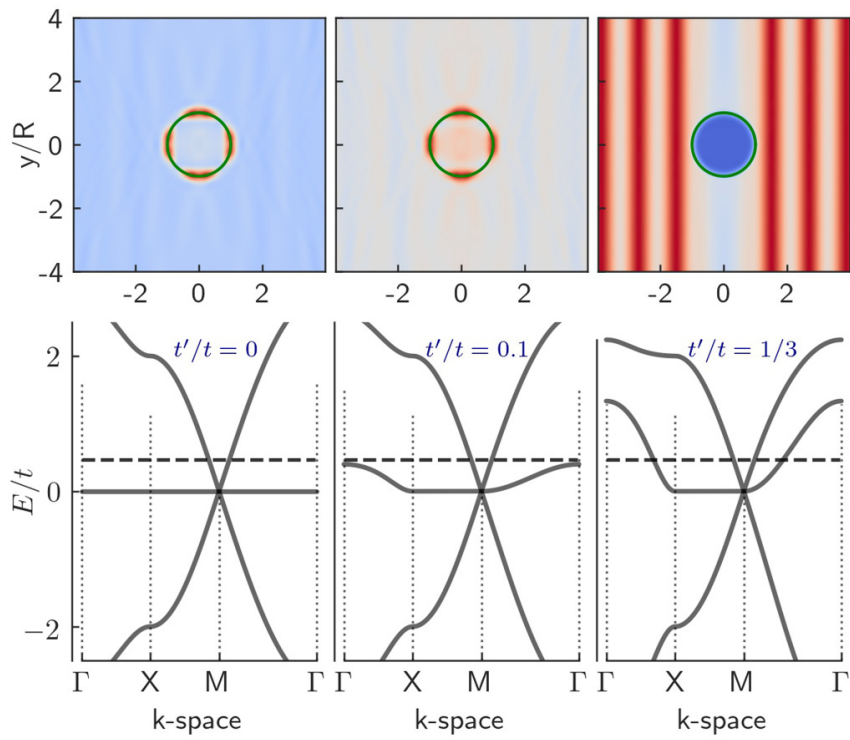

FIG. 6. Effect of the next-nearest-neighbor hopping $t^{\prime}$ in the tight-binding Lieb lattice based simulation on the localized surface mode analog in the Klein tunneling regime. Shown are the modes for $t^{\prime} / t=0$ (top left), $t^{\prime} / t=0.1$ (top middle), and $t^{\prime} / t=1 / 3$ (top right). The bottom panels are the corresponding band diagrams. Other parameters used are $V_{0} / t=1$ and $E / t=0.47$ (i.e., $E \sim V_{0} / 2$, as predicted by our theory, marked by the horizontal dashed lines in the bottom panels).

avenue for localized plasmon mimetic nanoelectronics, where gate-controlled localization or confinement and manipulation of quasiparticle quantum states are desirable in constructing functional circuitry and quantum computing devices. The finding enables realization of spoof localized plasmons in broader contexts than previously thought $[46,57,58]$.

References [4-8] reported the spin aspect of light through a Dirac- or Weyl-type description. However, our results are different. In particular, the results in Refs. [4-6] were restricted to a free and homogeneous space in constructing a proper Dirac-type matrix form of the Maxwell's equations, and Refs. [7,8] dealt with spin properties of propagating surface plasmons by drawing an analogy between the plasmonic states at the vacuum-metal interface and the surface states of the gapped Dirac spin-1/2 system at the domain wall separating regions with a sign-changing Dirac mass. Such Dirac surface states have actually been known for a long time and have been well studied using the Dirac equation for massive spin-1/2 particles $[59,60]$. Note that both the gapped nature of the massive Dirac system and the negative permittivity of a metal enable imaginary wave numbers to emerge and, hence, evanescent waves to naturally set in.

The analogy that we have uncovered is between the localized surface plasmon resonance modes (nonpropagating excitations) and those resulting from Klein scattering physics in gapless Dirac spin-1 systems, rather than gapped Dirac spin-1/2 systems. The gapless nature of the Dirac system leaves no room for imaginary wave numbers and the resulting evanescent channels especially in the Klein tunneling and subwavelength regime, where the picture of ray tracing based on total internal reflection is not applicable. As a result, the emergence of localized surface plasmon mimetic surface Dirac modes via Klein scattering is surprising in terms of the known Dirac theories on surface states and Klein tunneling. Counterintuitively, this analogy indicates that it is not necessary that creating evanescentlike surface modes involve a complex wave number due either to gap opening or to negative permittivity, as stipulated by the conventional wisdom.

It is worth emphasizing that the analogy uncovered in this paper creates a route to spoof surface plasmons without the well-documented need for any metal element or complicated texturing surfaces $[46,58,61,62]$. It also opens up the possibility of generating surface plasmon mimetic Dirac surface modes beyond the known gap-opening scenario in Dirac material systems as revealed in Ref. [7]. The analogy is counterintuitive and has unveiled an aspect or twist of the Klein paradox for spin-1 Dirac particles: It has the hidden "power" to generate localized surface plasmons. Our finding thus represents a fundamental contribution to our understanding of the physics of Klein tunneling and surface plasmons.

Another point is that the phenomenon of the field enhancement effect associated with localized surface waves on a spatial scale smaller than the wavelength in spin-1 Dirac systems arises from the coupled Klein scattering mechanism. Conventional wisdom in fact stipulates that Klein tunneling makes the boundary or interface more transparent with markedly high transmission probabilities passing through a classically forbidden region via waves that are not of the evanescent type [30]. This known and conventional feature of Klein tunneling has been exploited for applications such as focusing Dirac electron flow analogous to a transparent lens in classical optics [15]. In a resonator geometry, it is commonly believed that Klein tunneling is irrelevant to the surface modes, let alone localized surface plasmons in the subwavelength regime. Our finding goes beyond the common beliefs. Our results from the more intricate dimer geometries or structures without the circularly rotational symmetry (Fig. 3) provide further support for the unconventional analogy uncovered and have practical implication to simulating spoof plasmonic molecules with spin-1 Dirac-Weyl waves via multiple Klein scattering interactions.

Taken together, our finding paves the way for the interplay between localized surface plasmon subwavelength optics and Dirac spin-1 physics to mutually benefit both fields, which is counterintuitive with respect to existing knowledge.

\section{ACKNOWLEDGMENTS}

We would like to acknowledge support from the Vannevar Bush Faculty Fellowship program sponsored by the Basic Research Office of the Assistant Secretary of Defense for Research and Engineering and funded by the Office of Naval Research through Grant No. N00014-16-1-2828. L.H. is supported by NSFC under Grants No. 11775101 and No. 12047501, and by the 111 Project under Grant No. B20063

\section{APPENDIX A: DERIVATIONS OF EQUATIONS (2) AND (4)}

For the spin-1 Dirac-Weyl waves, the Mie-type solutions contain a series of partial scattering coefficients $\tilde{a}_{l}$ expressed 

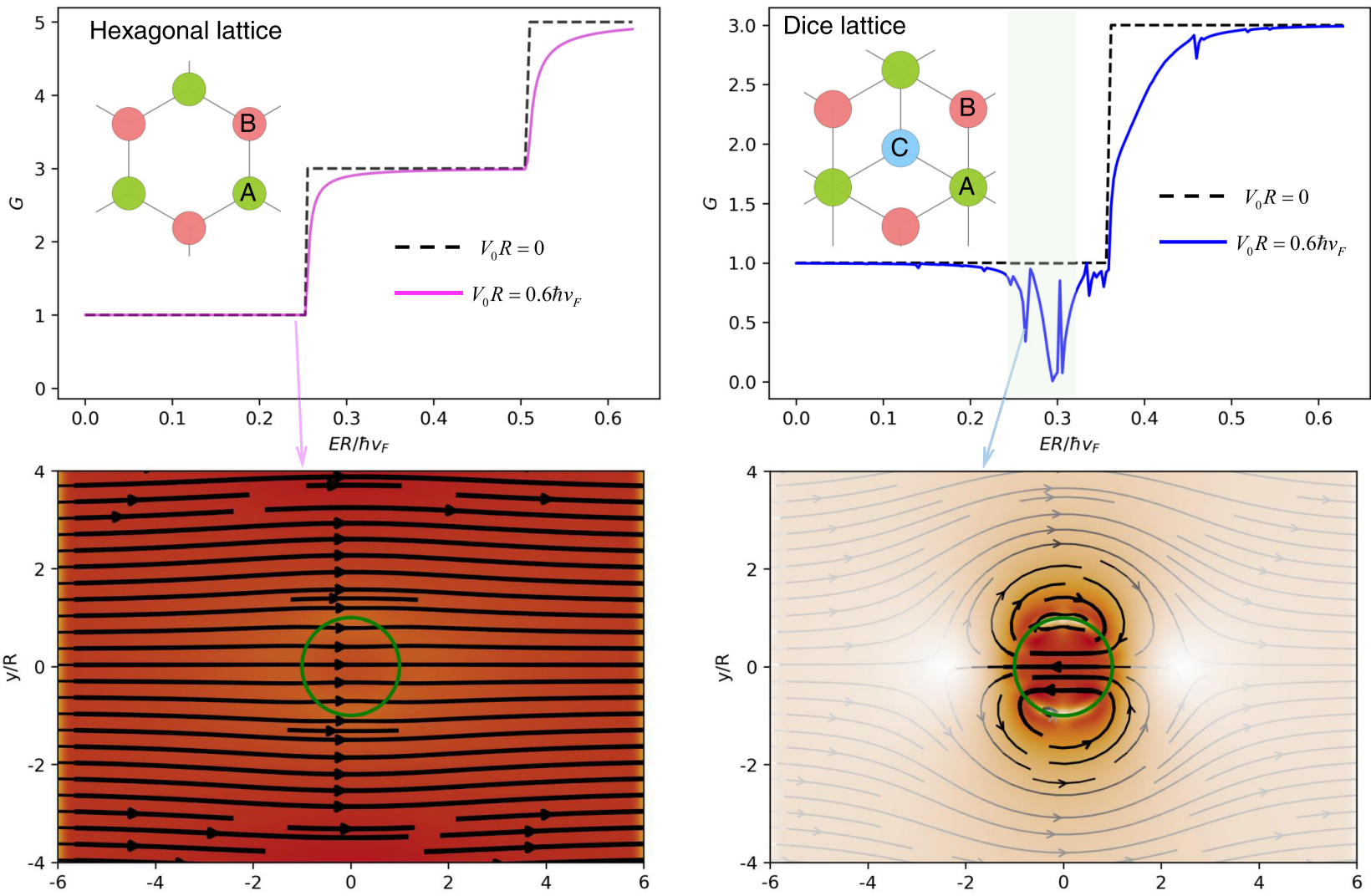

FIG. 7. Edge states arising from a hexagonal and a dice lattice. The left panels show the energy spectrum of the hexagonal lattice system that hosts pseudospin-1/2 quasiparticles. The right panels show the energy spectrum and edge states of the dice lattice consisting of two nested hexagonal lattices. While both dice and hexagonal lattices possess the same first Brillouin zone, the former has three sublattices and hosts a low-energy gapless spin-1 Dirac cone near each of the two nonequivalent valleys $K$ and $K^{\prime}$.

in terms of Bessel functions of integer order $l$ :

$$
\tilde{a}_{l}=-\frac{\tilde{F}_{l}}{\tilde{F}_{l}+i \tilde{G}_{l}},
$$

with

$$
\begin{gathered}
\tilde{F}_{l}=J_{l}(\eta \rho) J_{l}^{\prime}(\rho)-s_{I} s_{O} J_{l}^{\prime}(\eta \rho) J_{l}(\rho), \\
\tilde{G}_{l}=J_{l}(\eta \rho) Y_{l}^{\prime}(\rho)-s_{I} s_{O} J_{l}^{\prime}(\eta \rho) Y_{l}(\rho),
\end{gathered}
$$

where the normalized scatterer size is $\rho=k R \equiv E R /\left(\hbar v_{F}\right)$ and $\eta \equiv\left|\left(E-V_{0}\right) / E\right|$ is a parameter with $E$ being the particle kinetic energy measured from the Dirac point outside the scattering potential barrier. The band indices inside and outside the scatterer are labeled by $s_{I}=\operatorname{sgn}(E)$ and $s_{O}=$ $\operatorname{sgn}\left(E-V_{0}\right)$, respectively, and $J_{l}$ and $Y_{l}$ are the Bessel functions of the first and second kinds, while $\mathcal{Z}_{l}^{\prime} \equiv d \mathcal{Z}_{l}(z) / d z$ for $\mathcal{Z}_{l} \in\left\{J_{l}, Y_{l}\right\}$.

By definition, the scattering efficiency is given by

$$
\tilde{\Lambda}_{\text {scat }} \equiv \frac{\tilde{\sigma}}{2 R}=\frac{2}{\rho} \sum_{l=-\infty}^{\infty}\left|\tilde{a}_{l}\right|^{2} .
$$

In the regime of small scatterer size $\rho \ll 1$ (i.e., the subwavelength regime $R \ll \lambda / 2 \pi$ with $\lambda$ being the wavelength of the incoming spin-1 Dirac-Weyl particles), we have the leading terms in the expressions of $\tilde{F}_{l}$ and $\tilde{G}_{l}$ as

$$
\begin{aligned}
& \tilde{F}_{l} \approx \begin{cases}\frac{s_{l} s_{o} \eta-1}{2} \rho+\cdots, & \text { for } l=0 \\
\frac{\eta^{l-1}\left[\eta-s_{l} s_{o}\right]}{2 l !(l-1) !}\left(\frac{\rho}{2}\right)^{2 l-1}+\cdots & \text { for } l>0,\end{cases} \\
& \tilde{G}_{l} \approx \begin{cases}\frac{2}{\pi \rho}+\cdots & \text { for } l=0 \\
\frac{\eta^{l-1}\left[\eta+s_{l} s_{O}\right]}{\pi \rho}+\cdots & \text { for } l>0 .\end{cases}
\end{aligned}
$$

Keeping the dominant terms up to $|l|=1$ for small $\rho$, we obtain Eqs. (2) and (3).

We next review the corresponding results on the scattering of an electromagnetic wave from a metallic cylinder. Consider an incident monochromatic plane wave of TM polarization propagating along the $x$ axis, with the magnetic field amplitude given by $\boldsymbol{H}^{\text {inc. }}=\hat{z} H_{z}^{\text {inc. }}=\hat{z} e^{i k x}$ (with the timeharmonic factor $e^{-i \omega t}$ dropped). The exact Mie solution of the Maxwell's equations gives the partial expansion coefficient as [34]

$$
a_{l}=-\frac{F_{l}}{F_{l}+i G_{l}},
$$

with

$$
\begin{aligned}
F_{l} & =\sqrt{\epsilon} J_{l}(\sqrt{\epsilon} \rho) J_{l}^{\prime}(\rho)-J_{l}^{\prime}(\sqrt{\epsilon} \rho) J_{l}(\rho), \\
G_{l} & =\sqrt{\epsilon} J_{l}(\sqrt{\epsilon} \rho) Y_{l}^{\prime}(\rho)-J_{l}^{\prime}(\sqrt{\epsilon} \rho) Y_{l}(\rho),
\end{aligned}
$$



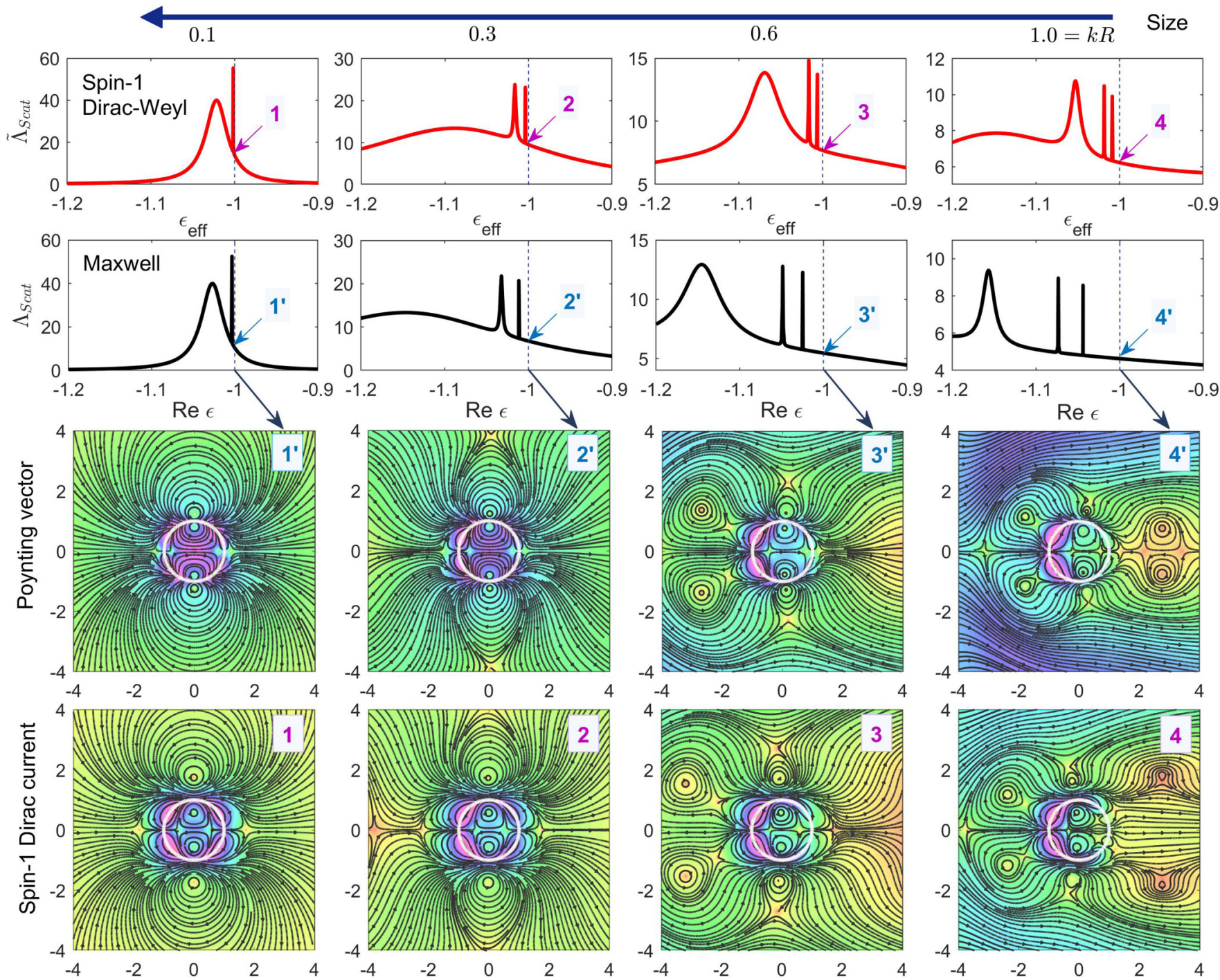

FIG. 8. Comparison of scattering line shape and related spatial patterns of flow or current. Top two rows: calculated scattering spectra for different scatterer sizes. Third and fourth rows: spatial distributions of the Poynting vector field and Dirac current at the corresponding parameter values indicated by the arrows in the top two rows. The black arrowed streamlines denote the flows of the Poynting vector or Dirac current. The color is scaled by magnitude.

where $\epsilon=\epsilon^{\prime}+i \epsilon^{\prime \prime}$ denotes the relative dielectric permittivity of the scatterer. It was obtained in previous work $[35,36]$ that for $\rho \ll 1$, the leading terms in the expressions of $F_{l}$ and $G_{l}$ are

$$
\begin{aligned}
& F_{l} \approx \begin{cases}\frac{\sqrt{\epsilon}(\epsilon-1)}{2}\left(\frac{\rho}{2}\right)^{3}+\cdots & \text { for } l=0 \\
\frac{(\sqrt{\epsilon})^{l-1}(\epsilon-1)}{2 l !(l-1) !}\left(\frac{\rho}{2}\right)^{2 l-1}+\cdots & \text { for } l>0,\end{cases} \\
& G_{l} \approx \begin{cases}\frac{2 \sqrt{\epsilon}}{\pi \rho}+\cdots & \text { for } l=0 \\
\frac{(\sqrt{\epsilon})^{l-1}(\epsilon+1)}{\pi \rho}+\cdots & \text { for } l>0 .\end{cases}
\end{aligned}
$$

We obtain the scattering efficiency as in Eq. (4).

\section{APPENDIX B: MORE RESULTS ON DIRAC CURRENTS AND POYNTING VECTORS}

We present an extended and more detailed comparison between the Dirac currents and Poynting vectors to reinforce the analogy between Dirac spin-1 and surface plasmon physics. In particular, Figs. 8 and 9 present the comparison results with varying scatterer size and across a particular resonance, respectively.

\section{APPENDIX C: MULTIPLE MULTIPOLE EXPANSION METHOD FOR SOLVING THE SCATTERING OF A SPIN-1 DIRAC-WEYL WAVE FROM A DIMER OF ARBITRARY SHAPE}

The multiple multipole expansion method originated from optics [63-67] and was adopted for photonic crystal waveguides [68] and more recently to Dirac-Weyl spinor systems under various circumstances [69-71].

To treat the scattering of a pseudospin-1 Dirac wave from a complicated coupled structure, we develop a multiple multipole method. To be concrete, we consider a dimer configuration of potential step of an arbitrary shape. There 

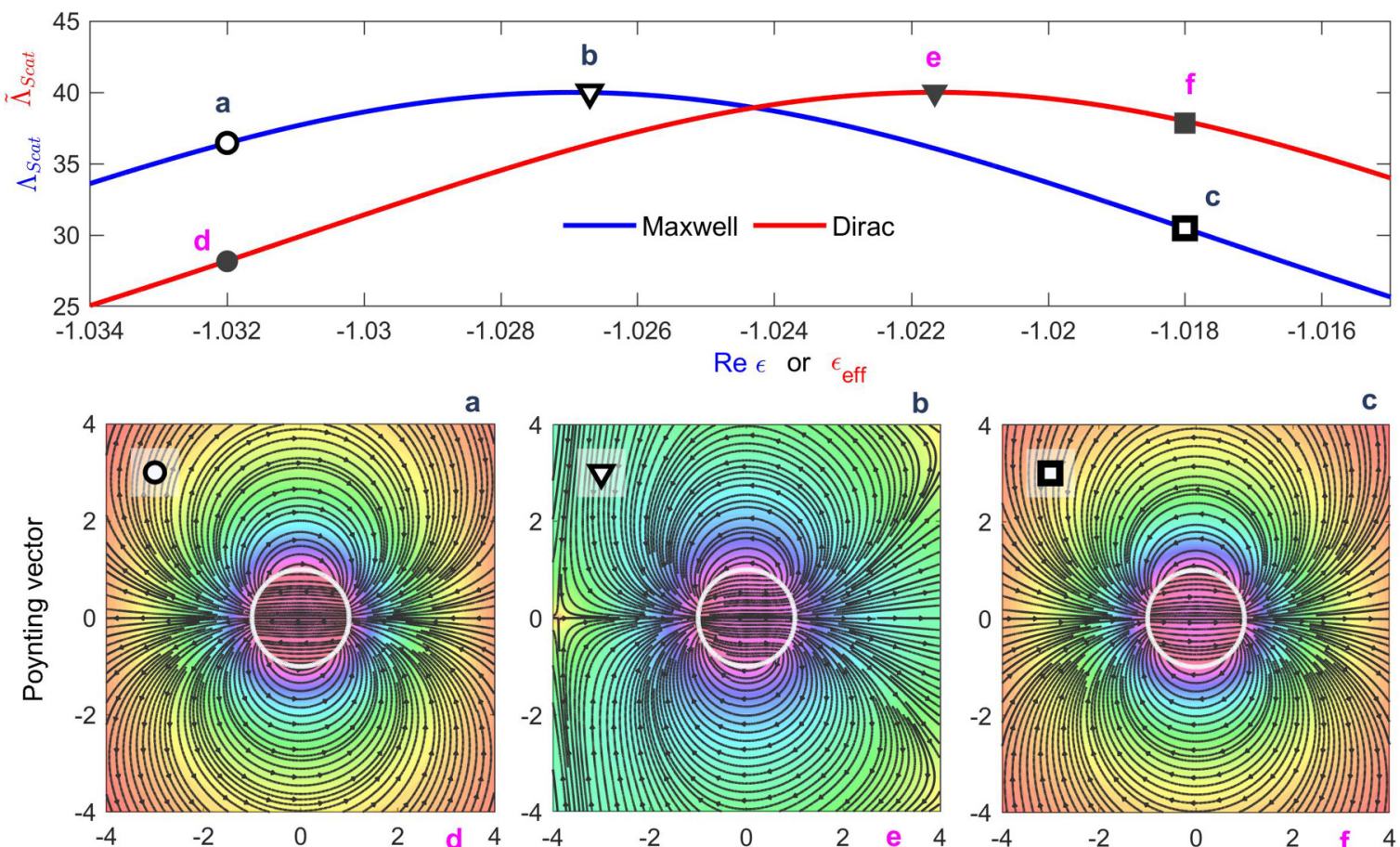

b
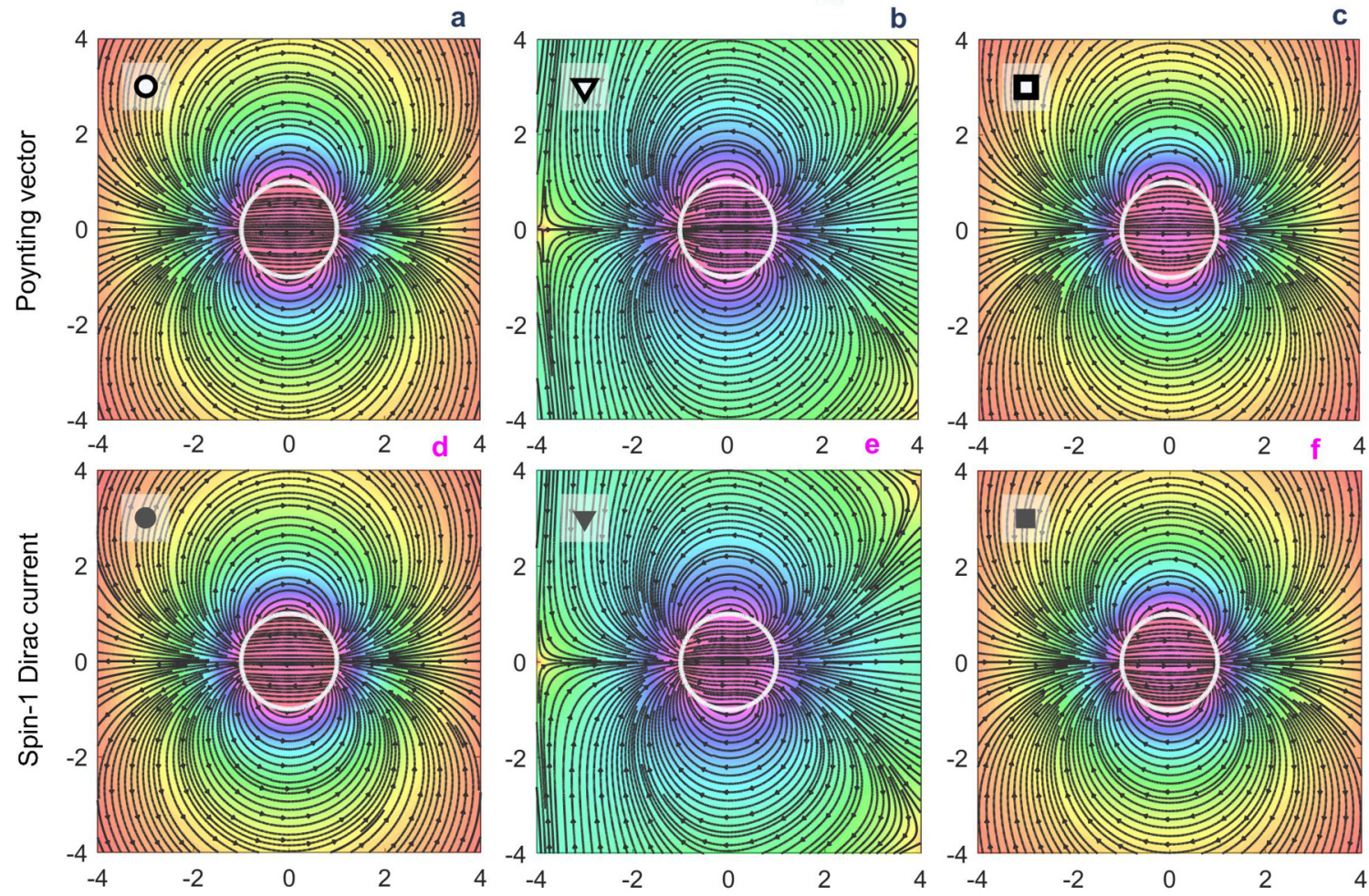

FIG. 9. Comparison of spatial pattern evolution of Poynting vector and Dirac current about a given resonance. Top panel: zoom-in on the dipole localized surface plasmon resonance (blue) and the first Klein scattering resonance (red) displayed in Fig. 1 of the main text. (a)-(c) show the spatial profiles of Poynting vector fields corresponding to the scattering states indicated in the top panel. (d)-(f) are for the case of a spin-1 Dirac wave.

are three subregions, denoted as I, II, and III, as shown in Fig. 10. The generalized Dirac-Weyl equation in each subregion $\tau \in\{\mathrm{I}, \mathrm{II}, \mathrm{III}\}$ is given by

$$
\hat{\boldsymbol{S}} \cdot \hat{\boldsymbol{k}} \Psi^{(\tau)}(\boldsymbol{r})=\epsilon_{\tau} \Psi^{(\tau)}(\boldsymbol{r}),
$$

where $\epsilon_{\tau}=\left(E-V_{\tau}\right) / \hbar v_{F}$. In the polar coordinates $r=(r, \theta)$, the spinor cylindrical wave basis of the solutions with angular momentum $l$ is

$$
\Psi_{l}^{(\tau)}(\boldsymbol{r})=\left(\begin{array}{c}
B_{l-1}\left(k_{\tau} r\right) e^{-i \theta} \\
i s_{\tau} B_{l}\left(k_{\tau} r\right) \\
-B_{l+1}\left(k_{\tau} r\right) e^{i \theta}
\end{array}\right) e^{i l \theta},
$$

where $s_{\tau}=\operatorname{sgn}\left(E-V_{\tau}\right)$ denotes the pertinent band index and $k_{\tau}=\left|E-V_{\tau}\right| / \hbar v_{F}$. Assuming $E>0$ and choosing $B_{l}\left(k_{\tau} r\right)=H_{l}^{(1)}\left(k_{\tau} r\right)$ (with $H_{l}^{(1)}$ being the Hankel function of the first kind), we define the Dirac-type wave functions of multipoles outside the specific solving region $\tau$ and positioned at $\boldsymbol{r}_{m_{\bar{\tau}}}$ as

$$
\Psi_{l}^{(\tau)}\left(\boldsymbol{d}_{m_{\bar{\tau}}}\right)=\frac{1}{\sqrt{2}}\left(\begin{array}{c}
H_{l-1}^{(1)}\left(k_{\tau} d_{m_{\bar{\tau}}}\right) e^{-i \theta_{m_{\bar{\tau}}}} \\
i \sqrt{2} s_{\tau} H_{l}^{(1)}\left(k_{\tau} d_{m_{\bar{\tau}}}\right) \\
-H_{l+1}^{(1)}\left(k_{\tau} d_{m_{\bar{\tau}}}\right) e^{i \theta_{m_{\bar{\tau}}}}
\end{array}\right) e^{i l \theta_{m_{\bar{\tau}}}},
$$

where $\bar{\tau}$ denotes the complement of $\tau$ and $d_{m_{\bar{\tau}}} \equiv\left|\boldsymbol{d}_{m_{\bar{\tau}}}\right|=$ $\left|\boldsymbol{r}-\boldsymbol{r}_{m_{\bar{\tau}}}\right|$ and $\theta_{m_{\bar{\tau}}}=\operatorname{Angle}\left(\boldsymbol{r}-\boldsymbol{r}_{m_{\bar{\tau}}}\right)$ with $\boldsymbol{r} \in \tau$. Carrying out multiple multipole expansion for the specific region, we obtain the wave function in region III as 


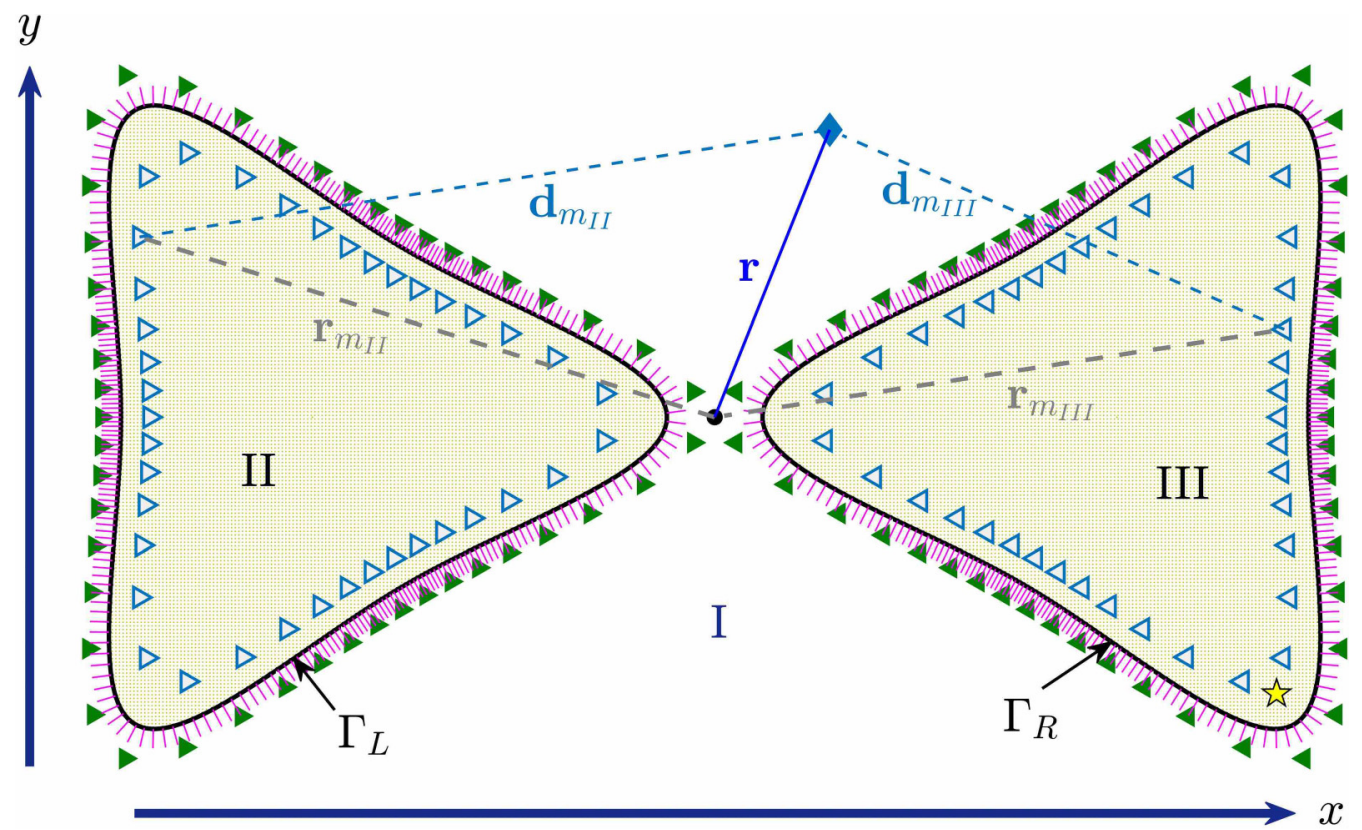

FIG. 10. Schematic illustration of multiple multipole expansion method. The physical boundary separating regions II and III is denoted as $\Gamma_{L / R}$, while the multiple multipoles ("fictitious" sources) are placed at $\boldsymbol{r}_{m_{\tau}}$ with $\tau \in\{$ I, II, III $\}$. The sources inside regions II and III (light-blue triangles) "radiate" pseudospin-1 Dirac wave field $\Psi_{l}^{(\mathrm{I})}\left(\boldsymbol{r}-\boldsymbol{r}_{m_{\mathrm{I} / \mathrm{III}}}\right)$, which is used to determine the wave function in region I. The sources in domain I (green triangles) generate the fields $\Psi_{l}^{(\mathrm{II})}\left(\boldsymbol{r}-\boldsymbol{r}_{m_{\mathrm{I}}}\right)$ and $\Psi_{l}^{(\mathrm{III})}\left(\boldsymbol{r}-\boldsymbol{r}_{m_{\mathrm{I}}}\right)$, which are used to determine the wave functions in regions II and III, respectively. Boundary conditions are satisfied at the collocation positions on $\Gamma_{L / R}$ (indicated by the short magenta lines).

$$
\Psi^{(\mathrm{III})}(\boldsymbol{r})=\sum_{m_{\mathrm{III}}} \sum_{l} C_{l}^{m_{\mathrm{III}}} \frac{1}{\sqrt{2}}\left(\begin{array}{l}
H_{l-1}^{(1)}\left(k_{\mathrm{III}} d_{m_{\mathrm{II}}}\right) e^{-i \theta_{m_{\mathrm{III}}}} \\
i \sqrt{2} s_{\mathrm{III}} H_{l}^{(1)}\left(k_{\mathrm{III}} d_{m_{\mathrm{III}}}\right) \\
-H_{l+1}^{(1)}\left(k_{\mathrm{III}} d_{m_{\mathrm{III}}}\right) e^{i \theta_{m_{\mathrm{III}}}}
\end{array}\right) e^{i l \theta_{m_{\mathrm{III}}}} \equiv\left(\begin{array}{c}
\psi_{1}^{\mathrm{III}} \\
\psi_{2}^{\mathrm{III}} \\
\psi_{3}^{\text {III }}
\end{array}\right) .
$$

The wave function in region II is obtained as

$$
\Psi^{(\mathrm{II})}(\boldsymbol{r})=\sum_{m_{\overline{\mathrm{I}}}} \sum_{l} B_{l}^{m_{\overline{\mathrm{I}}}} \frac{1}{\sqrt{2}}\left(\begin{array}{c}
H_{l-1}^{(1)}\left(k_{\mathrm{II}} d_{m_{\bar{I}}}\right) e^{-i \theta_{m_{\bar{\Pi}}}} \\
i \sqrt{2} s_{\mathrm{II}} H_{l}^{(1)}\left(k_{\mathrm{II}} d_{m_{\overline{\mathrm{I}}}}\right) \\
-H_{l+1}^{(1)}\left(k_{\mathrm{II}} d_{m_{\bar{\Pi}}}\right) e^{i \theta_{\overline{\mathrm{II}}}}
\end{array}\right) e^{i l \theta_{m_{\bar{\Pi}}}} \equiv\left(\begin{array}{c}
\psi_{1}^{\mathrm{II}} \\
\psi_{2}^{\mathrm{II}} \\
\psi_{3}^{\mathrm{II}}
\end{array}\right) .
$$

The scattered (outgoing) wave function in region I has the form

$$
\Psi^{(\mathrm{I})}(\boldsymbol{r})=\sum_{m_{\overline{\mathrm{I}}}} \sum_{l} A_{l}^{m_{\overline{\mathrm{T}}}} \frac{1}{\sqrt{2}}\left(\begin{array}{c}
H_{l-1}^{(1)}\left(k_{\mathrm{I}} d_{m_{\overline{\mathrm{T}}}}\right) e^{-i \theta_{m_{\mathrm{T}}}} \\
i \sqrt{2} s_{\mathrm{I}} H_{l}^{(1)}\left(k_{\mathrm{I}} d_{m_{\overline{\mathrm{I}}}}\right) \\
-H_{l+1}^{(1)}\left(k_{\mathrm{I}} d_{m_{\mathrm{I}}}\right) e^{i \theta_{m_{\mathrm{T}}}}
\end{array}\right) e^{i l \theta_{m_{\mathrm{I}}}} \equiv\left(\begin{array}{c}
\psi_{1}^{\mathrm{I}} \\
\psi_{2}^{\mathrm{I}} \\
\psi_{3}^{\mathrm{I}}
\end{array}\right) .
$$

A planar incident wave propagating along the direction that makes an angle $\beta$ with the $x$ axis in region I can be written as

$$
\Psi^{\text {in }}(\boldsymbol{r})=\frac{1}{2}\left(\begin{array}{c}
e^{-i \beta} \\
\sqrt{2} s_{\mathrm{I}} \\
e^{i \beta}
\end{array}\right) e^{i k \cdot r}=\left(\begin{array}{c}
\psi_{1}^{\text {in }} \\
\psi_{2}^{\text {in }} \\
\psi_{3}^{\text {in }}
\end{array}\right)
$$

Imposing the boundary conditions parametrized by the angle $\alpha$ between the outward normal at any boundary point $\boldsymbol{r}_{j}$ and the $x$ axis,

$$
\begin{gathered}
\left.\left(\psi_{2}^{(\mathrm{I})}+\psi_{2}^{\mathrm{in}}\right)\right|_{r_{j} \in \Gamma_{v}}=\left.\psi_{2}^{(\tau)}\right|_{\boldsymbol{r}_{j} \in \Gamma_{v}}, \\
\left.\left(\left[\psi_{1}^{(\mathrm{I})}+\psi_{1}^{\mathrm{in}}\right] e^{i \alpha}+\left[\psi_{3}^{(\mathrm{I})}+\psi_{3}^{\mathrm{in}}\right] e^{-i \alpha}\right)\right|_{\boldsymbol{r}_{j} \in \Gamma_{v}}=\left.\left(\psi_{1}^{(\tau)} e^{i \alpha}+\psi_{3}^{(\tau)} e^{-i \alpha}\right)\right|_{\boldsymbol{r}_{j} \in \Gamma_{v}},
\end{gathered}
$$


with $v=L, R$, we obtain

$$
\begin{aligned}
& \sum_{m_{\mathrm{II}}} \sum_{l}{ }^{j} A_{l m_{\mathrm{II}}}^{(\mathrm{I})} C_{l}^{m_{\mathrm{II}}}-\sum_{m_{\mathrm{I}}} \sum_{l}{ }^{j} A_{l m_{\mathrm{I}}}^{(\mathrm{II})} C_{l}^{m_{\mathrm{I}}}=-{ }^{j} \psi_{2}^{\mathrm{in}}, \\
& \sum_{m_{\mathrm{II}}} \sum_{l}{ }^{j} B_{l m_{\mathrm{II}}}^{(\mathrm{I})} C_{l}^{m_{\mathrm{II}}}-\sum_{m_{\mathrm{I}}} \sum_{l}{ }^{j} B_{l m_{l}}^{(\mathrm{II})} C_{l}^{m_{\mathrm{I}}}=-{ }^{j} \chi^{\mathrm{in}},
\end{aligned}
$$

where the substitutions are given by

$$
\begin{aligned}
{ }^{j} A_{l m_{\mathrm{II}}}^{(\mathrm{I})} & =i s_{\mathrm{I}} H_{l}^{(1)}\left(k_{\mathrm{I}}\left|\boldsymbol{r}_{j}-\boldsymbol{r}_{m_{\mathrm{II}}}\right|\right) e^{i l \theta_{m_{\mathrm{II}}}}, \\
{ }^{j} A_{l m_{\mathrm{I}}}^{(\mathrm{II})} & =i s_{\mathrm{II}} H_{l}^{(1)}\left(k_{\mathrm{II}}\left|\boldsymbol{r}_{j}-\boldsymbol{r}_{m_{\mathrm{I}}}\right|\right) e^{i l \theta_{m_{\mathrm{I}}},} \\
{ }^{j} B_{l m_{\mathrm{II}}}^{(\mathrm{I})} & =\frac{1}{\sqrt{2}}\left[H_{l-1}^{(1)}\left(k_{\mathrm{I}}\left|\boldsymbol{r}_{j}-\boldsymbol{r}_{m_{\mathrm{II}}}\right|\right) e^{i(l-1) \theta_{m_{\mathrm{II}}}} e^{i \alpha}-H_{l+1}^{(1)}\left(k_{\mathrm{I}}\left|\boldsymbol{r}_{j}-\boldsymbol{r}_{m_{\mathrm{II}}}\right|\right) e^{\left.i(l+1) \theta_{m_{\mathrm{II}}} e^{-i \alpha}\right],}\right. \\
{ }^{j} B_{l m_{\mathrm{I}}}^{(\mathrm{II})} & =\frac{1}{\sqrt{2}}\left[H_{l-1}^{(1)}\left(k_{\mathrm{II}}\left|\boldsymbol{r}_{j}-\boldsymbol{r}_{m_{\mathrm{I}}}\right|\right) e^{i(l-1) \theta_{m_{\mathrm{I}}}} e^{i \alpha}-H_{l+1}^{(1)}\left(k_{\mathrm{II}}\left|\boldsymbol{r}_{j}-\boldsymbol{r}_{m_{\mathrm{I}}}\right|\right) e^{i(l+1) \theta_{m_{\mathrm{I}}}} e^{-i \alpha}\right]
\end{aligned}
$$

and

$$
\begin{gathered}
{ }^{j} \psi_{2}^{\text {in }}=\frac{1}{\sqrt{2}} S_{\mathrm{I}} e^{i \boldsymbol{k}_{I} \cdot \boldsymbol{r}_{j}}, \\
{ }^{j} \chi^{\text {in }}=\frac{1}{2}\left[e^{i(\alpha-\beta)}+e^{-i(\alpha-\beta)}\right] e^{i \boldsymbol{k}_{I} \cdot \boldsymbol{r}_{j}} .
\end{gathered}
$$

In principle, the set consists of an infinite number of equations with an infinite number of undetermined expansion coefficients $C_{l}^{m_{\mathrm{II}}}$ and $C_{l}^{m_{\mathrm{I}}}$. To solve the system numerically, a finite truncation is necessary, which turns out to be feasible in practice by discretizing the boundary to a finite number of points $J$ and setting the number of multipoles $M_{\tau}$ in the specific region $\tau$ and $l \in[-L, L]$ for all the multipoles. Carrying out the discretization procedure, we arrive at the following finite-dimensional matrix equation:

$$
\mathbb{M}_{2 J \times N} \cdot C_{N \times 1}=-\boldsymbol{Y}_{2 J \times 1}
$$

where $N=(2 L+1) \times\left(M_{\mathrm{I}}+M_{\mathrm{II}}+M_{\mathrm{III}}\right)=N_{I}+N_{\mathrm{II}}+N_{\mathrm{III}}$ and the compact substitutions are

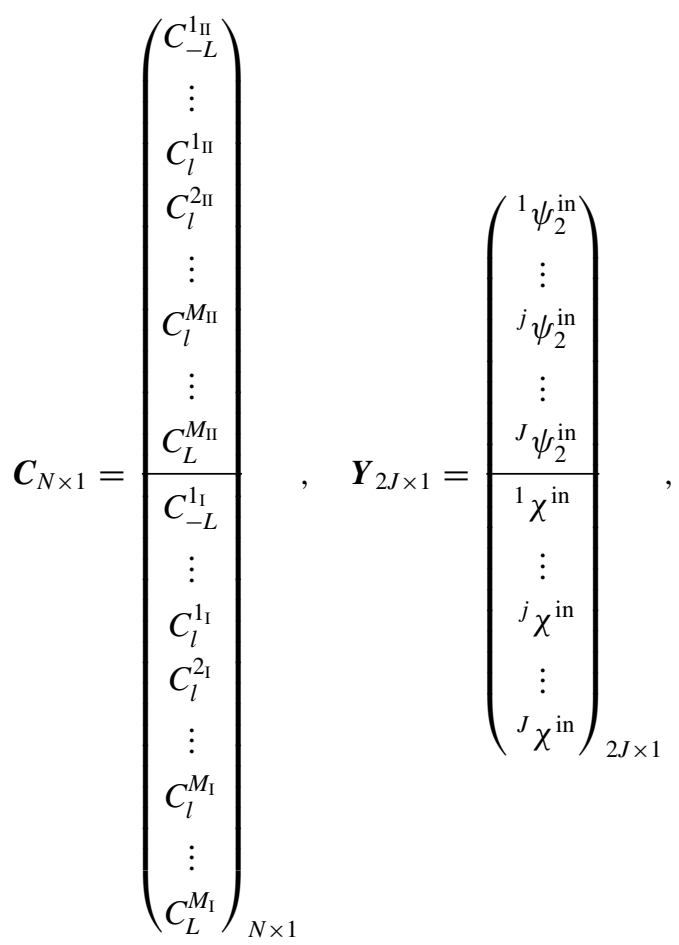


and

$$
\mathbb{M}_{2 J \times N}=\left(\begin{array}{c|c|c}
\mathbb{P}_{J_{I}}^{(\mathrm{I})} & -\mathbb{P}_{J_{\mathrm{I}}}^{(\mathrm{II})} & 0 \\
\hline \mathbb{Q}_{J_{\mathrm{I}}}^{(\mathrm{I})} & -\mathbb{Q}_{J_{I}}^{(\mathrm{II})} & 0 \\
\hline \mathbb{P}_{J_{\mathrm{II}}}^{(\mathrm{I})} & 0 & -\mathbb{P}_{J_{\mathrm{II}}}^{(\mathrm{III})} \\
\hline \mathbb{Q}_{J_{\text {II }}}^{(\mathrm{I})} & 0 & -\mathbb{Q}_{J_{\text {II }}}^{(\mathrm{III})}
\end{array}\right)_{2 J \times N},
$$

with

$$
\begin{aligned}
& \mathbb{P}_{J}^{(\tau)}=\left(\begin{array}{cccccccc}
{ }^{1} A_{-L 1_{\bar{\tau}}}^{(\tau)} & \ldots & { }^{1} A_{l 1_{\bar{\tau}}}^{(\tau)} & { }^{1} A_{l 2_{\bar{\tau}}}^{(\tau)} & \ldots & { }^{1} A_{l M_{\bar{\tau}}}^{(\tau)} & \ldots & { }^{1} A_{L M_{\bar{\tau}}}^{(\tau)} \\
{ }^{2} A_{-L 1_{\bar{\tau}}}^{(\tau)} & \ldots & { }^{2} A_{l 1_{\bar{\tau}}}^{(\tau)} & { }^{2} A_{l 2_{\bar{\tau}}}^{(\tau)} & \ldots & { }^{2} A_{l M_{\bar{\tau}}}^{(\tau)} & \ldots & { }^{2} A_{L M_{\bar{\tau}}}^{(\tau)} \\
\vdots & \ldots & \vdots & \vdots & \ldots & \vdots & \ldots & \vdots \\
{ }^{j} A_{-L 1_{\bar{\tau}}}^{(\tau)} & \ldots & { }^{j} A_{l 1_{\bar{\tau}}}^{(\tau)} & { }^{j} A_{l 2_{\bar{\tau}}}^{(\tau)} & \ldots & { }^{j} A_{l M_{\bar{\tau}}}^{(\tau)} & \ldots & { }^{j} A_{L M_{\bar{\tau}}}^{(\tau)} \\
\vdots & \ldots & \vdots & \vdots & \ldots & \vdots & \ldots & \vdots \\
{ }^{J} A_{-L 1_{\bar{\tau}}}^{(\tau)} & \ldots & { }^{J} A_{l 1_{\bar{\tau}}}^{(\tau)} & { }^{J} A_{l 2_{\bar{\tau}}}^{(\tau)} & \ldots & { }^{J} A_{l M_{\bar{\tau}}}^{(\tau)} & \ldots & { }^{J} A_{L M_{\bar{\tau}}}^{(\tau)}
\end{array}\right)_{J \times N_{\bar{\tau}}} \\
& \mathbb{Q}_{J}^{(\tau)}=\left(\begin{array}{cccccccc}
{ }^{1} B_{-L 1_{\bar{\tau}}}^{(\tau)} & \ldots & { }^{1} B_{l 1_{\bar{\tau}}}^{(\tau)} & { }^{1} B_{l 2_{\bar{\tau}}}^{(\tau)} & \ldots & { }^{1} B_{l M_{\bar{\tau}}}^{(\tau)} & \ldots & { }^{1} B_{L M_{\bar{\tau}}}^{(\tau)} \\
{ }^{2} B_{-L 1_{\bar{\tau}}}^{(\tau)} & \ldots & { }^{2} B_{l 1_{\bar{\tau}}}^{(\tau)} & { }^{2} B_{l 2_{\bar{\tau}}}^{(\tau)} & \ldots & { }^{2} B_{l M_{\bar{\tau}}}^{(\tau)} & \ldots & { }^{2} B_{L M_{\bar{\tau}}}^{(\tau)} \\
\vdots & \ldots & \vdots & \vdots & \ldots & \vdots & \ldots & \vdots \\
{ }^{j} B_{-L 1_{\bar{\tau}}}^{(\tau)} & \cdots & { }^{j} B_{l 1_{\bar{\tau}}}^{(\tau)} & { }^{j} B_{l 2_{\bar{\tau}}}^{(\tau)} & \ldots & { }^{j} B_{l M_{\bar{\tau}}}^{(\tau)} & \ldots & { }^{j} B_{L M_{\bar{\tau}}}^{(\tau)} \\
\vdots & \ldots & \vdots & \vdots & \ldots & \vdots & \ldots & \vdots \\
{ }^{J} B_{-L 1_{\bar{\tau}}}^{(\tau)} & \ldots & { }^{J} B_{l 1_{\bar{\tau}}}^{(\tau)} & { }^{J} B_{l 2_{\bar{\tau}}}^{(\tau)} & \ldots & { }^{J} B_{l M_{\bar{\tau}}}^{(\tau)} & \ldots & { }^{J} B_{L M_{\bar{\tau}}}^{(\tau)}
\end{array}\right)_{J \times N_{\bar{\tau}}}
\end{aligned}
$$

As the expansions are generally nonorthogonal, more equations are required than unknowns to enable deduction of an overdetermined matrix system with $2 J \gg N$, which can be solved by the pseudoinverse algorithm (e.g., in MATLAB): $\boldsymbol{C}=-\operatorname{pinv}(\mathbb{M}) * \boldsymbol{Y}$. In particular, we use the residual error evaluated at the boundary

$$
\mathrm{SSE}=\frac{\|\mathbb{M} * \boldsymbol{C}+\boldsymbol{Y}\|}{\|\boldsymbol{Y}\|}
$$

as the criterion for testing convergence (where SSE is sum of squared errors). We adjust the number, the order, and/or the positions of the multipoles to ensure SSE < tolerance. After the unknown coefficients $\boldsymbol{C}$ have been obtained, the associated wave functions and hence the local density of states in the specific region can be calculated accordingly.

[1] E. Yablonovitch, Inhibited Spontaneous Emission in SolidState Physics and Electronics, Phys. Rev. Lett. 58, 2059 (1987).

[2] S. John, Strong Localization of Photons in Certain Disordered Dielectric Superlattices, Phys. Rev. Lett. 58, 2486 (1987).

[3] E. Ruska, The development of the electron microscope and of electron microscopy, Rev. Mod. Phys. 59, 627 (1987).

[4] O. Laporte and G. E. Uhlenbeck, Application of spinor analysis to the Maxwell and Dirac equations, Phys. Rev. 37, 1380 (1931).

[5] J. R. Oppenheimer, Note on light quanta and the electromagnetic field, Phys. Rev. 38, 725 (1931).

[6] I. Bialynicki-Birula, $V$ photon wave function, in Progress in Optics, edited by E. Wolf (Elsevier, New York, 1996), Vol. 36, pp. 245-294.

[7] K. Y. Bliokh, D. Smirnova, and F. Nori, Quantum spin Hall effect of light, Science 348, 1448 (2015).

[8] S. A. R. Horsley, Topology and the optical Dirac equation, Phys. Rev. A 98, 043837 (2018).

[9] T. Van Mechelen and Z. Jacob, Quantum gyroelectric effect: Photon spin-1 quantization in continuum topological bosonic phases, Phys. Rev. A 98, 023842 (2018).
[10] V. M. Todd and J. Zubin, Unidirectional Maxwellian spin waves, Nanophotonics 8, 1399 (2019).

[11] T. V. Mechelen and Z. Jacob, Photonic Dirac monopoles and skyrmions: Spin-1 quantization, Opt. Mater. Express 9, 95 (2019).

[12] K. Y. Bliokh, D. Leykam, M. Lein, and F. Nori, Topological non-Hermitian origin of surface Maxwell waves, Nat. Commun. 10, 580 (2019).

[13] D. Jin, L. Lu, Z. Wang, C. Fang, J. D. Joannopoulos, M Soljaacic, L. Fu, and N. X. Fang, Topological magnetoplasmon, Nat. Commun. 7, 13486 (2016).

[14] P. Delplace, J. B. Marston, and A. Venaille, Topological origin of equatorial waves, Science 358, 1075 (2017).

[15] V. V. Cheianov, V. Fal'ko, and B. L. Altshuler, The focusing of electron flow and a Veselago lens in graphene $p$ - $n$ junctions, Science 315, 1252 (2007).

[16] J. Cserti, A. Pályi, and C. Péterfalvi, Caustics due to a Negative Refractive Index in Circular Graphene $p$ - $n$ Junctions, Phys. Rev. Lett. 99, 246801 (2007).

[17] R. L. Heinisch, F. X. Bronold, and H. Fehske, Mie scattering analog in graphene: Lensing, particle confinement, and depletion of Klein tunneling, Phys. Rev. B 87, 155409 (2013). 
[18] J. Caridad, S. Connaughton, C. Ott, H. B. Weber, and V. Krstic, An electrical analogy to Mie scattering, Nat. Commun. 7, 12894 (2016).

[19] C. W. J. Beenakker, R. A. Sepkhanov, A. R. Akhmerov, and J. Tworzydło, Quantum Goos-Hänchen Effect in Graphene, Phys. Rev. Lett. 102, 146804 (2009).

[20] Q.-D. Jiang, H. Jiang, H. Liu, Q.-F. Sun, and X. C. Xie, Topological Imbert-Fedorov Shift in Weyl Semimetals, Phys. Rev. Lett. 115, 156602 (2015).

[21] Y. Zhao, J. Wyrick, F. D. Natterer, J. F. Rodriguez-Nieva, C. Lewandowski, K. Watanabe, T. Taniguchi, L. S. Levitov, N. B. Zhitenev, and J. A. Stroscio, Creating and probing electron whispering-gallery modes in graphene, Science 348, 672 (2015).

[22] J. R. Williams, T. Low, M. S. Lundstrom, and C. M. Marcus, Gate-controlled guiding of electrons in graphene, Nat. Nanotechnol. 6, 222 (2011).

[23] A. Cheng, T. Taniguchi, K. Watanabe, P. Kim, and J.-D. Pillet, Guiding Dirac Fermions in Graphene with a Carbon Nanotube, Phys. Rev. Lett. 123, 216804 (2019).

[24] K. Wang, M. M. Elahi, L. Wang, K. M. M. Habib, T. Taniguchi, K. Watanabe, J. Hone, A. W. Ghosh, G.-H. Lee, and P. Kim, Graphene transistor based on tunable Dirac fermion optics, Proc. Natl. Acad. Sci. USA 116, 6575 (2019).

[25] D. Leykam and A. S. Desyatnikov, Conical intersections for light and matter waves, Adv. Phys.: X 1, 101 (2016).

[26] T. Ozawa, H. M. Price, A. Amo, N. Goldman, M. Hafezi, L. Lu, M. C. Rechtsman, D. Schuster, J. Simon, O. Zilberberg, and I. Carusotto, Topological photonics, Rev. Mod. Phys. 91, 015006 (2019).

[27] R. Shen, L. B. Shao, B. Wang, and D. Y. Xing, Single Dirac cone with a flat band touching on line-centered-square optical lattices, Phys. Rev. B 81, 041410(R) (2010).

[28] A. Fang, Z. Q. Zhang, S. G. Louie, and C. T. Chan, Klein tunneling and supercollimation of pseudospin-1 electromagnetic waves, Phys. Rev. B 93, 035422 (2016).

[29] P. Strange, Relativistic Quantum Mechanics: With Applications in Condensed Matter and Atomic Physics (Cambridge University Press, Cambridge, 1998) .

[30] M. Katsnelson, K. Novoselov, and A. Geim, Chiral tunnelling and the Klein paradox in graphene, Nat. Phys. 2, 620 (2006).

[31] C. W. J. Beenakker, Colloquium: Andreev reflection and Klein tunneling in graphene, Rev. Mod. Phys. 80, 1337 (2008).

[32] R. Yang, L. Huang, Y.-C. Lai, and C. Grebogi, Abnormal electron paths induced by Klein tunneling in graphene quantum point contacts, Phys. Rev. B 84, 035426 (2011).

[33] H.-Y. Xu and Y.-C. Lai, Revival resonant scattering, perfect caustics, and isotropic transport of pseudospin-1 particles, Phys. Rev. B 94, 165405 (2016).

[34] C. Bohren and D. Huffman, Absorption and Scattering of Light by Small Particles, Wiley Science Series (Wiley, New York, 2008).

[35] M. I. Tribelsky and B. S. Luk'yanchuk, Anomalous Light Scattering by Small Particles, Phys. Rev. Lett. 97, 263902 (2006).

[36] B. S. Luk'yanchuk and V. Ternovsky, Light scattering by a thin wire with a surface-plasmon resonance: Bifurcations of the Poynting vector field, Phys. Rev. B 73, 235432 (2006).

[37] M. V. Berry, Optical currents, J. Opt. A: Pure Appl. Opt. 11, 094001 (2009).
[38] L. Landau, J. Bell, M. Kearsley, L. Pitaevskii, E. Lifshitz, and J. Sykes, Electrodynamics of Continuous Media (Elsevier Science, New York, 2013).

[39] W. L. Barnes, A. Dereux, and T. W. Ebbesen, Surface plasmon subwavelength optics, Nature (London) 424, 824 (2003).

[40] V. Myroshnychenko, J. Rodríguez-Fernández, I. PastorizaSantos, A. M. Funston, C. Novo, P. Mulvaney, L. M. Liz-Marzán, and F. J. García de Abajo, Modelling the optical response of gold nanoparticles, Chem. Soc. Rev. 37, 1792 (2008).

[41] P. Nordlander, C. Oubre, E. Prodan, K. Li, and M. I. Stockman, Plasmon hybridization in nanoparticle dimers, Nano Lett. 4, 899 (2004).

[42] T. W. Ebbesen, C. Genet, and S. I. Bozhevolnyi, Surfaceplasmon circuitry, Phys. Today 61(5), 44 (2008).

[43] H. A. Atwater and A. Polman, Plasmonics for improved photovoltaic devices, Nat. Mater. 9, 205 (2010).

[44] J. A. Schuller, E. S. Barnard, W. Cai, Y. C. Jun, J. S. White, and M. L. Brongersma, Plasmonics for extreme light concentration and manipulation, Nat. Mater. 9, 193 (2010).

[45] S. Thongrattanasiri and F. J. García de Abajo, Optical Field Enhancement by Strong Plasmon Interaction in Graphene Nanostructures, Phys. Rev. Lett. 110, 187401 (2013).

[46] A. Pors, E. Moreno, L. Martin-Moreno, J. B. Pendry, and F. J. Garcia-Vidal, Localized Spoof Plasmons Arise while Texturing Closed Surfaces, Phys. Rev. Lett. 108, 223905 (2012).

[47] R. Drost, T. Ojanen, A. Harju, and P. Liljeroth, Topological states in engineered atomic lattices, Nat. Phys. 13, 668 (2017).

[48] M. R. Slot, T. S. Gardenier, P. H. Jacobse, G. C. P. van Miert, S. N. Kempkes, S. J. M. Zevenhuizen, C. M. Smith, D. Vanmaekelbergh, and I. Swart, Experimental realization and characterization of an electronic Lieb lattice, Nat. Phys. 13, 672 (2017).

[49] J.-P. Tetienne, N. Dontschuk, D. A. Broadway, A. Stacey, D. A. Simpson, and L. C. L. Hollenberg, Quantum imaging of current flow in graphene, Sci. Adv. 3, e1602429 (2017).

[50] X. Huang, Y. Lai, Z. H. Hang, H. Zheng, and C. T. Chan, Dirac cones induced by accidental degeneracy in photonic crystals and zero-refractive-index materials, Nat. Mater. 10, 582 (2011).

[51] R. A. Vicencio, C. Cantillano, L. Morales-Inostroza, B. Real, C. Mejía-Cortés, S. Weimann, A. Szameit, and M. I. Molina, Observation of Localized States in Lieb Photonic Lattices, Phys. Rev. Lett. 114, 245503 (2015).

[52] F. Diebel, D. Leykam, S. Kroesen, C. Denz, and A. S Desyatnikov, Conical Diffraction and Composite Lieb Bosons in Photonic Lattices, Phys. Rev. Lett. 116, 183902 (2016).

[53] W. Y. Deng, X. Q. Huang, J. Y. Lu, F. Li, J. H. Ma, S. Q. Chen, and Z. Y. Liu, Acoustic spin-1 Weyl semimetal, Sci. China: Phys., Mech. Astron. 63, 287032 (2020).

[54] S. Carr, C. Li, Z. Zhu, E. Kaxiras, S. Sachdev, and A. Kruchkov, Ultraheavy and ultrarelativistic Dirac quasiparticles in sandwiched graphenes, Nano Lett. 20, 3030 (2020).

[55] D. Takane, Z. Wang, S. Souma, K. Nakayama, T. Nakamura, H. Oinuma, Y. Nakata, H. Iwasawa, C. Cacho, T. Kim, K. Horiba, H. Kumigashira, T. Takahashi, Y. Ando, and T. Sato, Observation of Chiral Fermions with a Large Topological Charge and Associated Fermi-Arc Surface States in CoSi, Phys. Rev. Lett. 122, 076402 (2019).

[56] Z. Rao, H. Li, T. Zhang, S. Tian, C. Li, B. Fu, C. Tang, L. Wang, Z. Li, W. Ran, J. Li, Y. Huang, Z. Liu, Y. Long, C. Fang, H. Weng, Y. Shi, H. L. Lei, Y. Sun, T. Qian, and H. Ding, 
Observation of unconventional chiral fermions with long Fermi arcs in CoSi, Nature (London) 567, 496 (2019).

[57] A. Devilez, X. Zambrana-Puyalto, B. Stout, and N. Bonod, Mimicking localized surface plasmons with dielectric particles, Phys. Rev. B 92, 241412(R) (2015).

[58] X. Shen and T. J. Cui, Ultrathin plasmonic metamaterial for spoof localized surface plasmons, Laser Photonics Rev. 8, 137 (2014).

[59] R. Jackiw and C. Rebbi, Solitons with fermion number $\frac{1}{2}$, Phys. Rev. D 13, 3398 (1976).

[60] A. P. Schnyder, S. Ryu, A. Furusaki, and A. W. W. Ludwig, Classification of topological insulators and superconductors in three spatial dimensions, Phys. Rev. B 78, 195125 (2008)

[61] P. A. Huidobro, X. Shen, J. Cuerda, E. Moreno, L. MartinMoreno, F. J. Garcia-Vidal, T. J. Cui, and J. B. Pendry, Magnetic Localized Surface Plasmons, Phys. Rev. X 4, 021003 (2014).

[62] J. Zhang, Z. Liao, Y. Luo, X. Shen, S. A. Maier, and T. J. Cui, Spoof plasmon hybridization, Laser Photonics Rev. 11, 1600191 (2017).

[63] Y. Leviatan and A. Boag, Analysis of electromagnetic scattering from dielectric cylinders using a multifilament current model, IEEE Trans. Antennas Propag. 35, 1119 (1987).
[64] M. G. Imhof, Multiple multipole expansions for elastic scattering, J. Acoust. Soc. Am. 100, 2969 (1996).

[65] D. I. Kaklamani and H. T. Anastassiu, Aspects of the method of auxiliary sources (MAS) in computational electromagnetics, IEEE Antennas Propag. Mag. 44, 48 (2002).

[66] E. Moreno, D. Erni, C. Hafner, and R. Vahldieck, Multiple multipole method with automatic multipole setting applied to the simulation of surface plasmons in metallic nanostructures, J. Opt. Soc. Am. A 19, 101 (2002).

[67] G. Tayeb and S. Enoch, Combined fictitious-sourcesscattering-matrix method, J. Opt. Soc. Am. A 21, 1417 (2004).

[68] E. Moreno, D. Erni, and C. Hafner, Modeling of discontinuities in photonic crystal waveguides with the multiple multipole method, Phys. Rev. E 66, 036618 (2002).

[69] H.-Y. Xu and Y.-C. Lai, Pseudospin-1 wave scattering that defies chaos $Q$-spoiling and Klein tunneling, Phys. Rev. B 99, 235403 (2019).

[70] H.-Y. Xu and Y.-C. Lai, Anomalous chiral edge states in spin-1 Dirac quantum dots, Phys. Rev. Res. 2, 013062 (2020).

[71] C.-Z. Wang, H.-Y. Xu, and Y.-C. Lai, Scattering of Dirac electrons from a skyrmion: Emergence of robust skew scattering, Phys. Rev. Research 2, 013247 (2020). 